IP/BBSR/2003-06

hep-th/0303223

\title{
D-branes in PP-wave light cone string field theory
}

\author{
B. Chandrasekhar*, Alok Kumar ${ }^{\dagger}$ \\ Institute of Physics, Bhubaneswar 751 005, INDIA
}

\begin{abstract}
The cubic interaction vertex and the dynamical supercharges are constructed for open strings ending on D7-branes, in light-cone superstring field theory in PP-wave background. In this context, we write down the symmetry generators in terms of the relevant group structure: $S U(2) \times S U(2) \times S O(2) \times S O(2)$, originating from the eight transverse directions in the PP-wave background and use the expressions to explicitly construct the vertex at the level of stringy zero modes. The results are further generalized to include all the stringy excitations as well.
\end{abstract}

October 30, 2018

*chandra@iopb.res.in

${ }^{\dagger}$ kumar@iopb.res.in 


\section{Introduction}

Maldacena's conjecture [1] of the duality between Type-IIB superstring theory in $A d S_{5} \times S^{5}$ background and $\mathcal{N}=4$ super-Yang-Mills theory has gone through several nontrivial checks at the level of supergravity. However, in most of the AdS/CFT dualities, it is not possible to go beyond the supergravity approximation on the string side, making its usefulness limited. Recently, the authors of [2] elucidated, that there exists a new scaling limit, in which a particular subsector of the $\mathcal{N}=4$ super-Yang-Mills theory gets mapped to Type-IIB superstring theory in a PP-wave background. PP-wave backgrounds can be obtained by taking a 'Penrose limit' [3, $4,5]$ of the geometry near a null geodesic in $A d S_{5} \times S^{5}$ carrying a large angular momentum on the $S^{5}$. The metric is then given by,

$$
d s^{2}=2 d x^{+} d x^{-}+\sum_{I=1}^{8}\left(d x_{I} d x^{I}-\mu^{2} x_{I} x^{I}\left(d x^{+}\right)^{2}\right),
$$

with a constant RR-5 form flux,

$$
F_{+1234}=F_{+5678}=2 \mu,
$$

where $\mu$ is a scaling parameter of mass dimension one. It is also interesting to note that the background in (1.1)-(1.2), preserves maximal IIB supersymmetry [6]. What makes the new duality tractable, is the fact that string theory in this background is exactly solvable in light-cone GS formalism [7], despite the presence of a nonvanishing RR field. Using this fact, the authors of [2] gave a proposal to match the string states of this free world sheet theory, to operators characterizing a certain subsector of the $\mathcal{N}=4$ super-Yang-Mills theory. The proposal amounts to picking a $U(1)_{R}$ subgroup of the $S U(4)$ R-symmetry and constructing trace operators, with a large $U(1)_{R}$ charge $J$ and conformal dimension $\Delta$, which together scale as $\sqrt{N}$, keeping the difference $\Delta-J$ finite in the large $N$ limit. Subsequent developments included deducing the string interaction vertices from the three point correlation functions of the these BMN operators $[8,9,10,11,12,13,14,15,16,17,18,19,20,21,22,23,24,25]$. Then using light-cone string field theory approach one constructs cubic string interaction vertices in PP-wave background, corresponding to splitting or joining of closed strings [26, 27, 28, 29, 30, $31,32]$. In this manner, one verifies the duality even at the level of interactions.

A generalization of AdS/CFT duality to the one between defect CFT and AdS spaces has also been analyzed in the literature $[33,34,35,36,37,38,39$, 40]. Such dualities in general originate from intersecting configurations involving a D3 brane with other D-branes, when a decoupling limit is applied on this brane configuration. The conformal structure of the boundary theory follows from the fact that the gauge theory living on the intersection of the $D 3$ and $D p$-brane goes over in the decoupling limit to the one living on an $A d S_{n} \subset A d S_{5}$. Therefore one also has a conformal structure of the $(n-1)$-dimensional defect CFT, in addition

to the one coming from $A d S_{5}$, thus giving a much richer physical structure. Considering such 
an interesting application of $D$-branes to the duality between string and gauge theories, it is important to study $D$-branes as well as their interactions [41], in PP-wave backgrounds. In this context, various brane configurations in PP-wave background have been studied. The world sheet constructions for the free theory on these branes have been given in $[42,43,44,45,46]$. Also, several supergravity solutions and other aspects of various brane configurations in PPwave background have been studied in [44, 45, 47]. In addition, emergence of open strings from super-Yang-Mills theory has also been shown by the authors of $[48,49]$. They construct certain determinant and sub-determinant operators, which turn out to be the Yang-Mills descriptions of the $D$-brane states or giant gravitons, in the PP-wave background. In a recent work $[50,51]$, certain symmetry related branes have been found and are argued to correspond to the giant gravitons.

In view of the above dualities between open strings and super-Yang-Mills at the free theory level, it becomes important to check whether this holds when the interactions are incorporated. Motivated by the above results, in this paper, we construct the cubic interaction vertex, corresponding to joining of two open strings to give a third open string, on a D7-brane. To perform this exercise, we use the light-cone superstring field theory formalism [52] developed by Green and Schwarz in flat background $[53,54]$. In the case of flat space, the transverse $S O(8)$ symmetry of the theory was given up in [55] and one used the spinors of $S U(4)$ for certain technical advantages. In the PP-wave background, $S O(8)$ symmetry is broken to $S O(4) \times S O(4)$. D7-brane boundary conditions break this symmetry further. We therefore use appropriate decompositions of the space-time coordinates in terms of the relevant symmetry: $S U(2)_{L} \times S U(2)_{R} \times S O(2)_{1} \times S O(2)_{2}$ to write down vertices and symmetry generators (for appearance of similar group structure in different context in string theory see [56]).

This paper is organized as follows. In section-2, we review the world sheet construction for $D p$-branes in PP-wave background and give expressions for all the symmetry generators. In section-3, we discuss the basics of light cone string field theory following the work of Green and Schwarz and construct the cubic interaction vertex involving stringy zero modes. In section-4, interaction vertex for all the non-zero open string modes is derived analogously. In section-5, we present discussions and conclusions.

\section{Review of worldsheet construction}

\section{$2.1 \quad$ D7-brane worldsheet theory}

We start by giving the main results of the open string construction for the case with both Neumann and Dirichlet boundary conditions. We will restrict ourselves to the case of $D 7$-branes in this paper, although other $D$-branes can be discussed along similar lines. The relevant bosonic

degrees of freedom in the light-cone gauge Green-Schwarz formalism $[7,43]$ are $X^{I}, I=1, \ldots, 8$, 
which transforms as a vector $\mathbf{8}_{\mathbf{v}}$ of the transverse $S O(8)$. In addition, one has space-time fermionic degrees of freedom: $S^{1}, S^{2}$ transforming as positive chirality spinors $\mathbf{8}_{\mathbf{s}}$ under $S O(8)$. To describe a Dirichlet $p$-brane, we impose Neumann boundary conditions on $p-1$ coordinates and Dirichlet boundary conditions on the remaining transverse coordinates:

$$
\begin{aligned}
& \partial_{\sigma} X^{r}=0, \quad r=1, \ldots, p-1, \\
& \partial_{\tau} X^{r^{\prime}}=0, \quad r^{\prime}=p, \ldots, 8 .
\end{aligned}
$$

The class of $D$ branes that we consider here belong to the category of 'longitudinal' D-branes in the language of [40]. For such branes the light-cone bosonic coordinates : $X^{ \pm}$also satisfy the Neumann boundary conditions [43]. For the fermionic coordinates, the boundary condition is

$$
\left.S^{1}\right|_{\sigma=0, \pi|\alpha|}=\left.\Omega S^{2}\right|_{\sigma=0, \pi|\alpha|},
$$

where, as in flat space, $\Omega$ is a real (constant) matrix $\Pi_{k} \gamma^{k}$, with the product running over the Dirichlet directions. Since, we are interested in $D$-brane configurations that preserve sixteen supesymmetries, the choice of allowed $\Omega$ is constrained further by the following two conditions:

$$
\begin{aligned}
{[\Omega, \gamma] } & =0 \\
\Omega \Pi \Omega \Pi & =-1 .
\end{aligned}
$$

$\Pi=\gamma^{1234}$ in eqn.(2.3) is the matrix that defines the form of the mass term on the worlsheet for these spinors and is responsible for breaking the transverse $S O(8)$ symmetry down to $S O(4) \times$ $S O(4)[2]$. The mode expansion of the bosonic coordinates, satisfying the equations of motion and the boundary conditions in eqns.(2.1), (2.2) is given by [43]:

$$
\begin{aligned}
X^{r}(\sigma, \tau) & =x_{0}^{r} \cos m \tau+\frac{p_{0}^{r}}{m} \sin m \tau+i \sum_{n \neq 0} \frac{1}{\omega_{n}} \alpha_{n}^{r} e^{-i \omega_{n} \tau} \cos \frac{n \sigma}{|\alpha|} \\
X^{r^{\prime}}(\sigma, \tau) & =\sum_{n \neq 0} \frac{1}{\omega_{n}} \alpha_{n}^{r^{\prime}} e^{-i \omega_{n} \tau} \sin \frac{n \sigma}{|\alpha|}
\end{aligned}
$$

with

$$
\omega_{n}=\operatorname{sgn}(n) \sqrt{(n / \alpha)^{2}+m^{2}},
$$

and $m=\mu p^{+}$. For the consistency of the supersymmetry algebra, one chooses the zero mode of $X^{r^{\prime}}$ to be zero, which corresponds to a $D p$-brane stuck at the origin $X^{r^{\prime}}=0$. The mode expansion of the fermions are:

$$
\begin{aligned}
& S^{1}(\sigma, \tau)=\cos m \tau S_{0}-\sin m \tau \Omega \Pi S_{0}+\sum_{n \neq 0} c_{n}\left(\varphi_{n}^{1}(\sigma, \tau) \Omega S_{n}+i \rho_{n} \varphi_{n}^{2}(\sigma, \tau) \Pi S_{n}\right), \\
& S^{2}(\sigma, \tau)=\cos m \tau \Omega^{T} S_{0}-\sin m \tau \Pi S_{0}+\sum_{n \neq 0} c_{n}\left(\varphi_{n}^{2}(\sigma, \tau) S_{n}-i \rho_{n} \varphi_{n}^{1}(\sigma, \tau) \Pi \Omega S_{n}\right),
\end{aligned}
$$


where,

$$
\begin{aligned}
\phi_{n}^{1}=e^{-i\left(\omega_{n} \tau-\frac{n}{|\alpha|} \sigma\right)}, & \phi_{n}^{2}=e^{-i\left(\omega_{n} \tau+\frac{n}{|\alpha|} \sigma\right)}, \\
c_{n}=\left(1+\rho_{n}^{2}\right)^{-1 / 2}, & \rho_{n}=\frac{\omega_{n}-n /|\alpha|}{m} .
\end{aligned}
$$

The canonical momenta are:

$$
P^{I}=\frac{1}{2 \pi|\alpha|} \dot{X}^{I}, \quad I=1, \ldots, 8, \quad \mathcal{P}^{\mathcal{I} a}=\frac{i}{2 \pi|\alpha|} S^{\mathcal{I} a}, \quad \mathcal{I}=1,2
$$

and the canonical commutation relations are given in terms of various modes as:

$$
\begin{aligned}
& {\left[x_{0}^{r}, p_{0}^{s}\right]=i \delta^{r s}, \quad\left[\alpha_{n}^{I}, \alpha_{m}^{J}\right]=\frac{1}{2} \omega_{n} \delta_{m+n, 0} \delta^{I J},} \\
& \left\{S_{n}^{a}, S_{m}^{b}\right\}=\frac{1}{4} \delta_{n+m, 0} \delta^{a b} .
\end{aligned}
$$

For definiteness we now consider the case of a D7-brane extending along $(+-123456)$ directions. This configuration breaks the $S O(4) \times S O(4)$ symmetry of the PP-wave background further. Thus we have,

$$
S O(8) \quad \supset S U(2)_{L} \times S U(2)_{R} \times S O(2)_{1} \times S O(2)_{2}
$$

where the $S U(2)_{L}$ and $S U(2)_{R}$ have their origin along directions $X^{1}, \ldots, X^{4}$ and the $S O(2)_{1} \times$ $S O(2)_{2}$ come from $X^{5}, \ldots, X^{8}$. Under the embedding in eqn.(2.13) the spinor decomposes as:

$$
\mathbf{8}_{\mathrm{s}} \sim(\mathbf{2}, \mathbf{1})^{\left(\frac{1}{2}, \frac{1}{2}\right)} \oplus(\overline{\mathbf{2}}, \mathbf{1})^{\left(-\frac{1}{2},-\frac{1}{2}\right)} \oplus(\mathbf{1}, 2)^{\left(\frac{1}{2},-\frac{1}{2}\right)} \oplus(1, \overline{2})^{\left(-\frac{1}{2}, \frac{1}{2}\right)}
$$

with the superscripts denoting $S O(2)_{1} \times S O(2)_{2}$ charges. Hence, the $S O(8)$ spinors decomposed in terms of the fermionic creation and annihilation operators are:

$$
\begin{array}{ll}
\bar{\lambda}_{\alpha}=S_{0 \alpha}^{\left(\frac{1}{2}, \frac{1}{2}\right)}, & \lambda_{\alpha}=S_{0 \alpha}^{\left(-\frac{1}{2},-\frac{1}{2}\right)} \\
\bar{\lambda}_{\dot{\alpha}}=S_{0 \dot{\alpha}}^{\left(-\frac{1}{2}, \frac{1}{2}\right)}, & \lambda_{\dot{\alpha}}=S_{0 \dot{\alpha}}^{\left(\frac{1}{2},-\frac{1}{2}\right)}
\end{array}
$$

where $\alpha$ and $\dot{\alpha}$ are the doublet indices of $S U(2)_{L}$ and $S U(2)_{R}$ respectively. Although, the decompositions (2.15) are given only for the zero modes in eqn.(2.7), similar results hold for the higher modes as well. The canonical anti-commutation relations in this basis take the form:

$$
\left\{\bar{\lambda}_{\alpha}, \lambda^{\beta}\right\}=\delta_{\alpha}^{\beta}, \quad\left\{\bar{\lambda}_{\dot{\alpha}}, \lambda^{\dot{\beta}}\right\}=\delta_{\dot{\alpha}}^{\dot{\beta}}
$$

The Fock vacuum is,

$$
a_{0}|0\rangle=0, \quad \lambda^{\alpha}|0\rangle=0, \quad \lambda^{\dot{\alpha}}|0\rangle=0 .
$$

Among the nonzero modes, the positive ones act as annihilation operators and the negative ones act as creation operators. 


\subsection{Symmetries of the free theory}

We now write the supersymmetry algebra and give the expressions for the symmetry generators for the free theory. In the light-cone formalism, the generators of the basic superalgebra can be split into the kinematical generators $\hat{P}^{+1}, \quad P^{I}, J^{+I}, J^{i j}, J^{i^{\prime} j^{\prime}}, Q_{a}^{+}, \bar{Q}_{a}^{+}$, and the dynamical ones $H, Q_{\dot{\alpha}}^{-1}, Q_{\dot{\alpha}}^{-2}$. The dynamical symmetry generators depend on all the non-zero stringy modes and also receive corrections from the interactions. As has been already mentioned, the left over isometry of the PP-wave due to the presence of $D 7$-branes is $S U(2)_{L} \times S U(2)_{R} \times$ $S O(2)_{1} \times S O(2)_{2}$, and the unbroken kinematical symmetriy generators are given as [7, 43, 50]:

$$
\begin{aligned}
\hat{P}^{+} & =p^{+}, \quad P^{r}=p_{0}^{r}, \quad J^{+r}=-x_{0}^{r} p^{+}, \\
Q^{+} & =\sqrt{2 p^{+}}\left(1+i \Omega^{T}\right) S_{0}, \quad \bar{Q}^{+}=\sqrt{2 p^{+}}\left(1-i \Omega^{T}\right) S_{0}, \\
J^{r s} & =x_{0}^{r} p_{0}^{s}-x_{0}^{s} p_{0}^{r}-i S_{0} \gamma^{r s} S_{0}-i \sum_{n \neq 0}\left\{\frac{1}{2 \omega_{n}}\left(\alpha_{-n}^{r} \alpha_{n}^{s}-\alpha_{-n}^{s} \alpha_{n}^{r}\right)+S_{-n} \gamma^{r s} S_{n}\right\}, \\
J^{r^{\prime} s^{\prime}} & =-i S_{0} \gamma^{r^{\prime} s^{\prime}} S_{0}-i \sum_{n \neq 0}\left\{\frac{1}{2 \omega_{n}}\left(\alpha_{-n}^{r^{\prime}} \alpha_{n}^{s^{\prime}}-\alpha_{-n}^{s^{\prime}} \alpha_{n}^{r^{\prime}}\right)+S_{-n} \gamma^{r^{\prime} s^{\prime}} S_{n}\right\}, \\
2 p^{+} H & =\frac{1}{2}\left(p_{0 r}^{2}+m^{2} x_{0 r}^{2}\right)-m i S_{0} \Omega \Pi S_{0}+\sum_{n \neq 0}\left\{\frac{1}{2} \alpha_{-n}^{I} \alpha_{n}^{I}+2 \omega_{n} S_{-n} S_{n}\right\}, \\
\sqrt{2 p^{+}} Q^{-1} & =\frac{1}{2 \pi \alpha^{\prime} p^{+}} \int_{0}^{2 \pi \alpha^{\prime}\left|p^{+}\right|} d \sigma\left(\partial_{-} X^{I} \gamma^{I} S^{1}-m X_{I} \gamma^{I} \Pi S^{2}\right), \\
& =p_{0}^{r} \gamma^{r} S_{0}+m x_{0}^{r} \gamma^{r} \Omega \Pi S_{0}-\sum_{n \neq 0}\left\{c_{n} \alpha_{-n}^{I} \Omega \gamma^{I} S_{n}-\frac{i m}{2 c_{n} \omega_{n}} \alpha_{-n}^{I} \gamma^{I} \Pi S_{n}\right\}, \\
\sqrt{2 p^{+}} Q^{-2} & =\frac{1}{2 \pi \alpha^{\prime} p^{+}} \int_{0}^{2 \pi \alpha^{\prime}\left|p^{+}\right|} d \sigma\left(\partial_{+} X^{I} \gamma^{I} S^{2}+m X_{I} \gamma^{I} \Pi S^{1}\right), \\
& =p_{0}^{r} \gamma^{r} \Omega^{T} S_{0}+m x_{0}^{r} \gamma^{r} \Pi S_{0}+\sum_{n \neq 0}\left\{c_{n} \alpha_{-n}^{I} \gamma^{I} S_{n}-\frac{i m}{2 c_{n} \omega_{n}} \alpha_{-n}^{I} \Omega^{T} \gamma^{I} \Pi S_{n}\right\},
\end{aligned}
$$

Since, $Q^{+}$and $\bar{Q}^{+}$as well as $Q^{-1}$ and $Q^{-2}$ are related as [43],

$$
Q^{+}+Q^{-}+i \Omega\left(Q^{+}-Q^{-}\right)=0, \quad Q^{-1}-\Omega Q^{-2}=0,
$$

one generally considers the particular combinations given below, which preserve the $D 7$-brane supersymmetries that we are interested in:

$$
\begin{aligned}
& q^{+}=\frac{1}{2}\left(Q^{+}+Q^{-}-i \Omega\left(Q^{+}-Q^{-}\right)\right)=2 \sqrt{2 p^{+}} S_{0}, \\
& q^{-}=Q^{-1}+\Omega Q^{-2}=2 Q^{-1} .
\end{aligned}
$$

${ }^{1}$ We have used a hat over the momentum generator to avoid mixup with components of momenta defined later on. 
The non-vanishing (anti-)commutation relations are [43]:

$$
\begin{aligned}
{\left[\hat{P}^{-}, P^{I}\right] } & =\mu^{2} J^{+I}, \quad\left[P^{I}, J^{+J}\right]=-\delta^{I J} \hat{P}^{+}, \\
{\left[\hat{P}^{-}, J^{+I}\right] } & =P^{I}, \quad\left[J^{r s}, q^{ \pm}\right]=\frac{i}{2} \gamma^{r s} q^{ \pm}, \\
{\left[J^{r^{\prime} s^{\prime}}, q^{ \pm}\right] } & =\frac{i}{2} \gamma^{r^{\prime} s^{\prime}} q^{ \pm}, \quad\left[J^{+r}, q^{-}\right]=\frac{i}{2} \Omega^{T} \gamma^{r} q^{+}, \\
{\left[P^{r}, q^{-}\right]=} & -i \frac{\mu}{2 p^{+}} \gamma^{r} \Pi q^{+}, \quad\left[H, q^{+}\right]=i \frac{\mu}{2 p^{+}} \Omega \Pi q^{+}, \\
\left\{q_{\alpha}^{+}, q_{\beta}^{+}\right\}= & \delta_{a b} 2 \hat{P}^{+}, \\
\left\{q_{\alpha}^{+}, q_{\dot{\alpha}}^{-}\right\}= & \left(\Omega \gamma^{r}\right)_{a \dot{\alpha}} P^{r}-\frac{\mu}{p^{+}}\left(\Pi \gamma^{r}\right)_{a \dot{\alpha}} J^{+r}, \\
\left\{q_{\dot{\alpha}}^{-}, q_{\dot{\beta}}^{-}\right\}= & \delta_{\dot{\alpha} \dot{\beta}} 2(H)+\frac{\mu}{2 p^{+}}\left(\left(\gamma_{I}^{r s} \Pi \Omega\right)_{\dot{\alpha} \dot{\beta}} J_{I}^{r s}+\left(\gamma_{I}^{r^{\prime} s^{\prime}} \Pi \Omega\right)_{\dot{\alpha} \dot{\beta}} J_{I}^{r^{\prime} s^{\prime}}\right) \\
& -\frac{\mu}{2 p^{+}}\left(\left(\gamma_{I I}^{r s} \Pi \Omega\right)_{\dot{\alpha} \dot{\beta}} J_{I I}^{r s}+\left(\gamma_{I I}^{r^{\prime} s^{\prime}} \Pi \Omega\right)_{\dot{\alpha} \dot{\beta}} J_{I I}^{r^{\prime} s^{\prime}}\right),
\end{aligned}
$$

where $J_{I}^{r s} \in S O(m), J_{I}^{r^{\prime} s^{\prime}} \in S O(4-m), J_{I I}^{r s} \in S O(n)$, and $J_{I I}^{r^{\prime} s^{\prime}} \in S O(4-n)$. In section-3, we will rewrite some of these generators and the commutation algebra using the symmetry structure in eqn.(2.13).

\section{Zero mode vertex in GS light-cone SFT}

In this section we first discuss some known results of the GS light-cone string field theory formalism and then apply them to write down the zero mode interaction vertex. As we shall see, most of the conceptual and technical aspects of the problem are already addressed in the zero mode analysis. We start by discussing the cubic open-string vertex corresponding to a process, where two open strings on a D7-brane join at their ends at $\tau=0$ to give a third open string. The construction follows the earlier work of Green and Schwarz [53, 54, 55] for flat space. It involves writing down the dynamical generators in terms of the string fields, which can create or destroy complete strings. The string fields can be expanded in a number basis representation as follows,

$$
\Phi[p(\sigma)]=\sum_{\left\{n_{k}\right\}} \varphi_{\left\{n_{k}\right\}} \prod_{k=-\infty}^{\infty} \psi_{n_{k}}\left(p_{k}\right),
$$

where $p_{k}$ is the $k$-th Fourier mode of $p(\sigma)$ and the sum is over all possible sets of harmonic oscillator occupation numbers $\left\{n_{k}\right\}$ with $\psi_{n}(p)$ being the harmonic oscillator wavefunction for occupation number $n$. Here, $\varphi_{\left\{n_{k}\right\}}$ is an operator that creates or destroys a string in the state $\left|\left\{n_{k}\right\}\right\rangle$ at time $\tau=0$. The full Hamiltonian has the form

$$
H=H_{2}+\kappa H_{3}+\ldots
$$


with similar expansions for $Q^{-}$and $\bar{Q}^{-}$, where $\kappa$ is the coupling constant. The interaction Hamiltonian as well as other dynamical generators can be expressed in terms of the string fields and hence, in a number basis representation. The coordinates of the three strings are parametrized as follows,

$$
\begin{array}{cccl}
\sigma_{(1)}= & \sigma & \text { for } & 0 \leq \sigma \leq \pi \alpha_{(1)}, \\
\sigma_{(2)}= & \sigma-\pi \alpha_{(1)} & \text { for } & \pi \alpha_{(1)} \leq \sigma \leq \pi\left(\alpha_{(1)}+\alpha_{(2)}\right), \\
\sigma_{(3)}= & \sigma-\pi\left(\alpha_{(1)}+\alpha_{(2)}\right) & \text { for } & 0 \leq \sigma \leq \pi\left(\alpha_{(1)}+\alpha_{(2)}\right),
\end{array}
$$

where $\alpha_{r}=2 \alpha^{\prime} p^{+}$, and $r=1,2,3$ denote the string indices . We also have $\alpha_{(1)}+\alpha_{(2)}+\alpha_{(3)}=0$, and one takes $\alpha_{(1)}, \alpha_{(2)}$ positive as the corresponding strings are annihilated by the vertex operator. With this labelling, the coordinates of the strings mean,

$$
X_{r}(\sigma)=x^{(r)}\left(\sigma_{r}\right) \Theta_{r}
$$

where

$$
\Theta_{(1)}=\theta\left(\pi \alpha_{(1)}-\sigma\right), \quad \Theta_{(2)}=\theta\left(\sigma-\pi \alpha_{(1)}\right), \quad \Theta_{(3)}=1
$$

The rest of the coordinates can be written in a similar form.

Before proceeding, it is important to decompose the $S O(8)$ invariant dynamical supercharges in the $S U(2)$ doublet notation according to the embedding (2.13). Under this embedding, the vectors decompose as,

$$
\mathbf{8}_{\mathbf{v}} \sim(\mathbf{2}, \mathbf{2})^{(0,0)} \oplus(\mathbf{1})^{(+1,0)} \oplus(\overline{\mathbf{1}})^{(-1,0)} \oplus(\mathbf{1})^{(0,+1)} \oplus(\overline{\mathbf{1}})^{(0,-1)}
$$

and the fermion decomposition is given in eqn.(2.14), where the superscripts indicate the $S O(2)_{1} \times S O(2)_{2}$ charges corresponding to rotations in 56 and 78 directions. To proceed further, we need various expressions written in terms of the representations of the group $S U(2)_{L} \times S U(2)_{R} \times S O(2)_{1} \times S O(2)_{2}$. Therefore, the relevant string degrees of freedom for us (e.g., eqn.(2.7)) are,

$$
S_{0}=\left(\begin{array}{c}
\lambda_{\dot{\alpha}}^{1} \\
\lambda_{\alpha}^{1} \\
\lambda_{\dot{\alpha}}^{2} \\
\lambda_{\alpha}^{2}
\end{array}\right)
$$

with $\lambda_{\alpha}=\lambda_{\alpha}^{1}-i \lambda_{\alpha}^{2}, \lambda_{\dot{\alpha}}=\lambda_{\dot{\alpha}}^{1}-i \lambda_{\dot{\alpha}}^{2}$ and $\lambda_{\alpha}, \lambda_{\dot{\alpha}}$ as given in eqn.(2.15). Similarly, in view of eqn.(3.6), the bosonic coordinates $X^{I}$ and and their conjugate momenta $P^{I}$ with $I=1, \ldots 8$, can be decomposed in terms of: $X_{\dot{\alpha} \alpha}, X^{ \pm}, \tilde{X}^{ \pm}$and $P_{\dot{\alpha} \alpha}, P^{ \pm}, \tilde{P}^{ \pm}$, where $X_{\dot{\alpha} \alpha}$ and $P_{\dot{\alpha} \alpha}$ are complex combinations of the coordinates $x^{1, \ldots 4}$ and $p^{1, \ldots 4}$ as given below:

$$
X_{\dot{\alpha} \alpha}=\left(\begin{array}{cc}
x^{4}+i x^{2} & -x^{3}+i x^{1} \\
x^{3}+i x^{1} & x^{4}-i x^{2}
\end{array}\right), \quad P_{\dot{\alpha} \alpha}=\left(\begin{array}{cc}
p^{4}+i p^{2} & -p^{3}+i p^{1} \\
p^{3}+i p^{1} & p^{4}-i p^{2}
\end{array}\right),
$$


with $\alpha$ and $\dot{\alpha}$ being the $S U(2)_{L}, S U(2)_{R}$ indices respectively. Further, we have the definitions, $X_{\alpha \dot{\alpha}}=X_{\dot{\alpha} \alpha}^{T}, \bar{X}_{\alpha \dot{\alpha}}=X_{\dot{\alpha} \alpha}^{\dagger}$, and similar ones for $P_{\alpha \dot{\alpha}}$ and $\bar{P}_{\alpha \dot{\alpha}}$. Also, $X^{ \pm}, \tilde{X}^{ \pm}$and $P^{ \pm}, \tilde{P}^{ \pm}$are defined as:

$$
\begin{array}{ll}
X^{ \pm}=x^{6} \pm i x^{5}, & \tilde{X}^{ \pm}=\tilde{x}^{8} \pm i \tilde{x}^{7}, \\
P^{ \pm}=p^{6} \pm i p^{5}, & \tilde{P}^{ \pm}=\tilde{p}^{8} \pm i \tilde{p}^{7} .
\end{array}
$$

Above combinations of coordinates and momenta have in fact been obtained by using a specific representation of 8-d Dirac $\gamma$ matrices:

$$
\begin{aligned}
& \gamma^{1}=\epsilon \otimes I \otimes \tau_{1}, \quad \gamma^{2}=\epsilon \otimes I \otimes \tau_{3}, \quad \gamma^{3}=I \otimes \tau_{3} \otimes \epsilon, \\
& \gamma^{4}=I \otimes I \otimes I, \quad \gamma^{5}=\epsilon \otimes \epsilon \otimes \epsilon, \quad \gamma^{6}=I \otimes \tau_{1} \otimes \epsilon, \\
& \gamma^{7}=\tau_{1} \otimes \epsilon \otimes I, \quad \gamma^{8}=\tau_{3} \otimes \epsilon \otimes I,
\end{aligned}
$$

where $\epsilon=i \tau_{2}$. These $\gamma^{\prime}$ 's which decompose the quantities $X^{I} \gamma^{I}$ and $P^{I} \gamma^{I}$ as in eqn.(3.8)-(3.10) are identical to the ones in [57], except for minor relabelling suitable to match with results in [43]. In this representation of $\gamma$ matrices $\Omega$ and $\Pi$ are given by,

$$
\Omega=\left(\begin{array}{cc}
0 & I_{4} \\
-I_{4} & 0
\end{array}\right), \quad \Pi=\left(\begin{array}{cc}
I_{2} \otimes \tau_{3} & 0 \\
0 & I_{2} \otimes \tau_{3}
\end{array}\right)
$$

Using the above results, supersymmetry generators are written in terms of $\lambda_{\alpha}, \lambda_{\dot{\alpha}}, \bar{\lambda}_{\alpha}, \bar{\lambda}_{\dot{\alpha}}$. The kinematical supersymmetry generators for example have the form:

$$
Q^{+}=\left(\begin{array}{c}
\bar{\lambda}_{\dot{\alpha}} \\
\bar{\lambda}_{\alpha} \\
-i \bar{\lambda}_{\dot{\alpha}} \\
-i \bar{\lambda}_{\alpha}
\end{array}\right), \quad \bar{Q}^{+}=\left(\begin{array}{c}
\lambda_{\dot{\alpha}} \\
\lambda_{\alpha} \\
i \lambda_{\dot{\alpha}} \\
i \lambda_{\alpha}
\end{array}\right)
$$

with invariant combinations in eqn.(2.28) given by eqn.(3.7). In eqn.(3.15), we have absorbed a factor of $\sqrt{2 p^{+}}$in the redefinition of $\lambda^{\prime}$ s, in order to make connection with results of [55].

Now, the procedure for constructing the vertex consists of two steps. The first step amounts to obtaining the kinematical part of the three string vertex that would occur, if the entire interaction Hamiltonian were given only by the kinematical factors and no other terms. These are the same as in flat space and arise from the commutation relations of dynamical generators $H$ with the kinematical generators $P^{I}, J^{+I}, q^{+}$. This gives the exponential part of the vertex. We postpone more detailed discussion of this part to section-4. Here, our main focus would be on the second step, which involves the calculation of prefactor, obtained from the algebra of dynamical generators.

At the level of zero modes, the kinematical part of the vertex takes a simple form and can be obtained by imposing the constraints coming from the PP-wave superalgebra of kinematical generators. Hence, the bosonic and fermionic coordinate continuity and local momentum 
conservation equations take the form:

$$
\begin{aligned}
\left(\alpha_{(1)} \hat{x}_{(1)}+\alpha_{(2)} \hat{x}_{(2)}+\alpha_{(3)} \hat{x}_{(3)}\right)\left|V_{k i n}\right\rangle & =0, \\
\left(\hat{p}_{(1)}+\hat{p}_{(2)}+\hat{p}_{(3)}\right)\left|V_{k i n}\right\rangle & =0, \\
\left(\alpha_{(1)} \hat{\lambda}_{(1)}+\alpha_{(2)} \hat{\lambda}_{(2)}+\alpha_{(3)} \hat{\lambda}_{(3)}\right)_{\alpha, \dot{\alpha}}\left|V_{k i n}\right\rangle & =0, \\
\left(\hat{\bar{\lambda}}_{(1)}+\hat{\bar{\lambda}}_{(2)}+\hat{\bar{\lambda}}_{(3)}\right)_{\alpha, \dot{\alpha}}\left|V_{k i n}\right\rangle & =0 .
\end{aligned}
$$

Note that the indices (which we have suppressed) on coordinates and momenta run only over the Neumann directions (having zero modes), and hence the constraints on the vertex are only in these directions.

Hence, the kinematical part of the zero mode vertex looks as follows:

$$
\left|V_{k i n}\right\rangle=\left|E_{b}^{0}\right\rangle\left|E_{f}^{0}\right\rangle
$$

where the bosonic part,

$$
\left|E_{b}^{0}\right\rangle \sim \exp \left[\frac{1}{2} \sum_{r, s=1}^{3} a_{(r)}^{\dagger} M^{r s} a_{(s)}^{\dagger}\right]|0\rangle
$$

and the fermionic part,

$$
\left|E_{f}^{0}\right\rangle \sim \frac{1}{4 !} \epsilon_{\alpha \beta} \epsilon_{\dot{\alpha} \dot{\beta}} \hat{\bar{\lambda}}^{\alpha} \hat{\bar{\lambda}}^{\beta} \hat{\bar{\lambda}}^{\dot{\alpha}} \hat{\bar{\lambda}}^{\dot{\beta}}|0\rangle,
$$

with $\hat{\bar{\lambda}}=\hat{\bar{\lambda}}_{(1)}+\hat{\bar{\lambda}}_{(2)}+\hat{\bar{\lambda}}_{(3)}$ and $a^{\dagger}=1 / \sqrt{\alpha \mu} \hat{p}_{0}+i / 2 \sqrt{\alpha \mu} \hat{x}_{0}$. The matrix $M^{r s}$ is as defined in [26], and is being given in the appendix. Having determined the kinematical part of the vertex $\left|V_{k i n}\right\rangle$, we now calculate the prefactor for the zero modes of the open strings ending on the $D 7$-brane.

The calculation of prefactor involves making an ansatz, for the functions multiplying the kinematical part of the vertex and the dynamical supercharges. The algebra of dynamical generators, then provides certain consistency conditions. These conditions determine the unknown functions uniquely. We begin by writing down the independent components of the dynamical supersymmetry generators, which can be seen by examining either $Q^{-1}$ or $Q^{-2}$. Using the decompositions (2.14) and (3.6), the dynamical supercharge given in eqn.(2.23) (or equivalently eqn.(2.29)) can be written in the following form:

$$
\begin{aligned}
& q_{\alpha}^{-}=\frac{1}{\alpha}\left[P_{\alpha \dot{\alpha}} \lambda_{\dot{\alpha}}+P^{+}(\epsilon \lambda)_{\alpha}\right]-i \mu\left[X_{\alpha \dot{\alpha}} \lambda_{\dot{\alpha}}-X^{+}(\epsilon \lambda)_{\alpha}\right], \\
& \bar{q}_{\alpha}^{-}=\frac{1}{\alpha}\left[\bar{P}_{\alpha \dot{\alpha}} \bar{\lambda}_{\dot{\alpha}}+P^{-}(\epsilon \bar{\lambda})_{\alpha}\right]+i \mu\left[\bar{X}_{\alpha \dot{\alpha}} \bar{\lambda}_{\dot{\alpha}}-X^{-}(\epsilon \bar{\lambda})_{\alpha}\right], \\
& q_{\dot{\alpha}}^{-}=\frac{1}{\alpha}\left[P_{\dot{\alpha} \alpha} \lambda_{\alpha}+P^{-}(\epsilon \lambda)_{\dot{\alpha}}\right]+i \mu\left[X_{\dot{\alpha} \alpha} \lambda_{\alpha}-X^{-}(\epsilon \lambda)_{\dot{\alpha}}\right], \\
& \bar{q}_{\dot{\alpha}}^{-}=\frac{1}{\alpha}\left[\bar{P}_{\dot{\alpha} \alpha} \bar{\lambda}_{\alpha}+P^{+}(\epsilon \bar{\lambda})_{\dot{\alpha}}\right]-i \mu\left[\bar{X}_{\dot{\alpha} \alpha} \bar{\lambda}_{\alpha}-X^{+}(\epsilon \bar{\lambda})_{\dot{\alpha}}\right] .
\end{aligned}
$$


Now, we use the above form of generators to determine the interaction vertex $|H\rangle,\left|q_{(r) \alpha}^{-}\right\rangle$etc., in a number basis defined in terms of the string fields as [55, 26]:

$$
\left\langle 3\left|H_{3}\right| 1\right\rangle|2\rangle=\langle 1|\langle 2|\langle 3 \mid H\rangle
$$

The kinematical part of the vertex has been already given in eqn.(3.20)-(3.22). The prefactors multiplying the kinematical vertex are given in terms of the unknown functions (to be determined below) $f_{\alpha \dot{\alpha}}, \bar{f}_{\alpha \dot{\alpha}}, f^{ \pm}, f_{\alpha}, f_{\dot{\alpha}}, \bar{f}_{\alpha}, \bar{f}_{\alpha}$ as:

$$
\begin{aligned}
|H\rangle & =\left[\mathbb{P}_{\alpha \dot{\alpha}} f^{\alpha \dot{\alpha}}+\overline{\mathbb{P}}_{\alpha \dot{\alpha}} \bar{f}^{\alpha \dot{\alpha}}+\mathbb{P}^{+} f^{-}+\mathbb{P}^{-} f^{+}\right]\left|V_{k i n}\right\rangle, \\
\left|q_{\alpha}^{-}\right\rangle & =f_{\alpha}\left|V_{k i n}\right\rangle, \\
\left|q_{\dot{\alpha}}^{-}\right\rangle & =f_{\dot{\alpha}}\left|V_{k i n}\right\rangle, \\
\left|\bar{q}_{\alpha}^{-}\right\rangle & =\bar{f}_{\alpha}\left|V_{k i n}\right\rangle, \\
\left|\bar{q}_{\dot{\alpha}}^{-}\right\rangle & =\bar{f}_{\dot{\alpha}}\left|V_{k i n}\right\rangle,
\end{aligned}
$$

where,

$$
\begin{aligned}
\mathbb{P}_{\dot{\alpha} \alpha} & =\alpha_{(1)} P_{\dot{\alpha} \alpha(2)}-\alpha_{(2)} P_{\dot{\alpha} \alpha(1)}, \\
\bar{\Lambda}_{\dot{\alpha}} & =\alpha_{(1)} \bar{\lambda}_{\dot{\alpha}(2)}-\alpha_{(2)} \bar{\lambda}_{\dot{\alpha}(1)},
\end{aligned}
$$

with similarly looking expressions for $\overline{\mathbb{P}}_{\alpha \dot{\alpha}}, P^{ \pm}, \Lambda_{\alpha}, \Lambda_{\dot{\alpha}}$ and $\bar{\Lambda}_{\alpha}$. The unknown quantities $f_{\alpha}, \bar{f}_{\alpha}$ etc., multiplying the vertex are assumed to be functions of only $\bar{\Lambda}$, as $\Lambda\left|V_{k i n}\right\rangle=0$. In other words, they are functions of the bosonic and fermionic conjugate momenta only. Above ansatz for the prefactors are slightly different from the ones in [54, 26] for the closed strings and are along the lines of results for open string in flat space [55]. The form of functions $f$ can now be determined by requiring that the PP-wave superalgebra is satisfied to $O(\kappa)$. For the generators $q_{(r) \alpha}^{-}$transforming nontrivially under the group $S U(2)_{L}$ in eqn.(2.36), this takes the form:

$$
\begin{aligned}
\sum_{r=1}^{3} q_{\alpha(r)}^{-}\left|\bar{q}_{\beta}^{-}\right\rangle+\sum_{r=1}^{3} \bar{q}_{\beta(r)}^{-}\left|q_{\alpha}^{-}\right\rangle & =2 \delta_{\alpha \beta}|H\rangle \\
\sum_{r=1}^{3} q_{\alpha(r)}^{-}\left|q_{\beta}^{-}\right\rangle+(\alpha \leftrightarrow \beta) & =0 \\
\sum_{r=1}^{3} \bar{q}_{\alpha(r)}^{-}\left|\bar{q}_{\beta}^{-}\right\rangle+(\alpha \leftrightarrow \beta) & =0 .
\end{aligned}
$$

One has identical relations for the $q_{\dot{\alpha}}^{\prime} s$ as well. The remaining ones between $q_{(r) \alpha}^{-}$and $q_{(r) \dot{\alpha}}^{-}{ }^{\prime} s$ are:

$$
\sum_{r=1}^{3} q_{\alpha(r)}^{-}\left|q_{\dot{\alpha}}^{-}\right\rangle+(\alpha \leftrightarrow \dot{\alpha})=0
$$




$$
\begin{aligned}
& \sum_{r=1}^{3} q_{\alpha(r)}^{-}\left|\bar{q}_{\dot{\alpha}}^{-}\right\rangle+(\alpha \leftrightarrow \dot{\alpha})=0, \\
& \sum_{r=1}^{3} \bar{q}_{\alpha(r)}^{-}\left|q_{\dot{\alpha}}^{-}\right\rangle+(\alpha \leftrightarrow \dot{\alpha})=0, \\
& \sum_{r=1}^{3} \bar{q}_{\alpha(r)}^{-}\left|\bar{q}_{\dot{\alpha}}^{-}\right\rangle+(\alpha \leftrightarrow \dot{\alpha})=0 .
\end{aligned}
$$

Also, the canonical commutation relations in our basis take the form:

$$
\begin{array}{lrl}
{\left[X_{\alpha \dot{\alpha}}, \bar{P}_{\beta \dot{\beta}}\right]=i \delta_{\alpha \beta} \delta_{\dot{\alpha} \dot{\beta}} \delta_{r s},} & {\left[\bar{X}_{\alpha \dot{\alpha}}, P_{\beta \dot{\beta}}\right]=i \delta_{\alpha \beta} \delta_{\dot{\alpha} \dot{\beta}} \delta_{r s},} \\
{\left[X^{ \pm}, P^{\mp}\right]=i \delta_{r s},} & {\left[\tilde{X}^{ \pm}, \tilde{P}^{\mp}\right]=i \delta_{r s},} \\
\left\{\bar{\lambda}_{\alpha(r)}, \lambda_{(s)}^{\beta}\right\}=\alpha_{r} \delta_{\alpha}^{\beta} \delta_{r s}, & \left\{\bar{\lambda}_{\left.\dot{\alpha}(r), \lambda_{(s)}^{\dot{\beta}}\right\}=\alpha_{r} \delta_{\dot{\alpha}}^{\dot{\beta}} \delta_{r s},}\right.
\end{array}
$$

with $\mathrm{r}$ and $\mathrm{s}$ being string indices. Further, one gets the following useful set of relations involving the supercharges $q_{(r) \alpha}^{-}$:

$$
\begin{aligned}
\sum_{r=1}^{3}\left[q_{(r) \alpha}^{-}, \overline{\mathbb{P}}_{\beta \dot{\beta}}\right] & =\mu \Lambda_{\dot{\alpha}} \delta_{\alpha \beta} \delta_{\dot{\alpha} \dot{\beta}} \\
\sum_{r=1}^{3}\left[q_{(r) \alpha}^{-}, \mathbb{P}^{-}\right] & =-\mu(\epsilon \Lambda)_{\alpha}, \\
\sum_{r=1}^{3}\left\{q_{(r) \alpha}^{-}, \bar{\Lambda}_{\beta}\right\} & =\left(\mathbb{P}^{+}-i \mu \alpha \mathbb{R}^{+}\right) \epsilon_{\alpha \beta}, \\
\sum_{r=1}^{3}\left\{q_{(r) \alpha}^{-}, \bar{\Lambda}_{\dot{\beta}}\right\} & =\left(\mathbb{P}_{\alpha \dot{\alpha}}+i \mu \alpha \mathbb{R}_{\alpha \dot{\alpha}}\right) \delta_{\dot{\alpha} \dot{\beta}},
\end{aligned}
$$

and the following algebra for the complex conjugates $\bar{q}_{(r) \alpha}^{-}$:

$$
\begin{aligned}
\sum_{r=1}^{3}\left[\bar{q}_{(r) \alpha}^{-}, \mathbb{P}_{\beta \dot{\beta}}\right] & =-\mu \bar{\Lambda}_{\dot{\beta}} \delta_{\alpha \beta}, \\
\sum_{r=1}^{3}\left[\bar{q}_{(r) \alpha}^{-}, \mathbb{P}^{+}\right] & =\mu(\epsilon \bar{\Lambda})_{\alpha}, \\
\sum_{r=1}^{3}\left\{\bar{q}_{(r) \alpha}^{-}, \Lambda_{\dot{\beta}}\right\} & =\left(\overline{\mathbb{P}}_{\alpha \dot{\alpha}}-i \mu \alpha \overline{\mathbb{R}}_{\alpha \dot{\alpha}}\right) \delta_{\dot{\alpha} \dot{\beta}}, \\
\sum_{r=1}^{3}\left\{\bar{q}_{(r) \alpha}^{-}, \Lambda_{\beta}\right\} & =\left(\mathbb{P}^{-}+i \mu \alpha \mathbb{R}^{-}\right) \epsilon_{\alpha \beta},
\end{aligned}
$$

where $\mathbb{R}_{\alpha \dot{\alpha}}=\frac{1}{\alpha_{3}}\left(X_{(2) \alpha \dot{\alpha}}-X_{(1) \alpha \dot{\alpha}}\right)$, with $\overline{\mathbb{R}}_{\alpha \dot{\alpha}}, \mathbb{R}^{ \pm}$defined analogously. Similarly, for the $q_{(r) \dot{\alpha}}^{-}{ }^{\prime} s$, we have:

$$
\sum_{r=1}^{3}\left[q_{(r) \dot{\alpha}}^{-}, \overline{\mathbb{P}}_{\dot{\beta} \beta}\right]=-\mu \Lambda_{\beta} \delta_{\dot{\alpha} \dot{\beta}}
$$




$$
\begin{aligned}
\sum_{r=1}^{3}\left[q_{(r) \dot{\alpha}}^{-}, \mathbb{P}^{+}\right] & =\mu(\epsilon \Lambda)_{\dot{\alpha}}, \\
\sum_{r=1}^{3}\left\{q_{(r) \dot{\alpha}}^{-}, \bar{\Lambda}_{\dot{\beta}}\right\} & =\left(\mathbb{P}^{-}+i \mu \alpha \mathbb{R}^{-}\right) \epsilon_{\dot{\alpha} \dot{\beta}}, \\
\sum_{r=1}^{3}\left\{q_{(r) \dot{\alpha}}^{-}, \bar{\Lambda}_{\beta}\right\} & =\left(\mathbb{P}_{\dot{\alpha} \alpha}-i \mu \alpha \mathbb{R}_{\dot{\alpha} \alpha}\right) \delta_{\alpha \beta} .
\end{aligned}
$$

The relations involving the complex conjugates $\bar{q}_{(r) \dot{\alpha}}^{-}$are given as:

$$
\begin{aligned}
\sum_{r=1}^{3}\left[\bar{q}_{(r) \dot{\alpha}}^{-}, \mathbb{P}_{\dot{\beta} \beta}\right] & =\mu \bar{\Lambda}_{\beta} \delta_{\dot{\alpha} \dot{\beta}}, \\
\sum_{r=1}^{3}\left[\bar{q}_{(r) \dot{\alpha}}^{-}, \mathbb{P}^{-}\right] & =-\mu(\epsilon \bar{\Lambda})_{\dot{\alpha}}, \\
\sum_{r=1}^{3}\left\{\bar{q}_{(r) \dot{\alpha}}^{-}, \Lambda_{\dot{\beta}}\right\} & =\left(\mathbb{P}^{+}-i \mu \alpha \mathbb{R}^{+}\right) \epsilon_{\dot{\alpha} \dot{\beta}}, \\
\sum_{r=1}^{3}\left\{\bar{q}_{(r) \dot{\alpha}}^{-}, \Lambda_{\beta}\right\} & =\left(\overline{\mathbb{P}}_{\dot{\alpha} \alpha}+i \mu \alpha \overline{\mathbb{R}}_{\dot{\alpha} \alpha}\right) \delta_{\alpha \beta} .
\end{aligned}
$$

Using the conservations laws in eqn.(3.16)-(3.19) the state $\left|V_{k i n}\right\rangle$ can be shown to satisfy:

$$
\begin{aligned}
\sum_{r=1}^{3} q_{(r) \alpha}^{-}\left|V_{k i n}\right\rangle & =0, \\
\sum_{r=1}^{3} q_{(r) \dot{\alpha}}^{-}\left|V_{\text {kin }}\right\rangle & =0, \\
\sum_{r=1}^{3} \bar{q}_{(r) \alpha}^{-}\left|V_{k i n}\right\rangle & =-\frac{1}{\alpha}\left[\overline{\mathbb{P}}_{\alpha \dot{\alpha}} \bar{\Lambda}_{\dot{\alpha}}+\mathbb{P}^{-}(\epsilon \bar{\Lambda})_{\alpha}\right]\left|V_{k i n}\right\rangle, \\
\sum_{r=1}^{3} \bar{q}_{(r) \dot{\alpha}}^{-}\left|V_{k i n}\right\rangle & =-\frac{1}{\alpha}\left[\overline{\mathbb{P}}_{\dot{\alpha} \alpha} \bar{\Lambda}_{\alpha}+\mathbb{P}^{+}(\epsilon \bar{\Lambda})_{\dot{\alpha}}\right]\left|V_{k i n}\right\rangle .
\end{aligned}
$$

Now, substituting our ansatz for the prefactors of the dynamical generators given in eqn.(3.28), in the PP-wave superalgebra in eqn.(3.36), (3.37) and using the above relations, we end up with the following equations for the unknown functions $f_{\alpha}, \bar{f}_{\alpha}$ :

$$
\begin{aligned}
& \frac{\partial f_{\beta}}{\partial \bar{\Lambda}_{\gamma}} \epsilon_{\alpha \gamma}+\frac{\partial f_{\alpha}}{\partial \bar{\Lambda}_{\gamma}} \epsilon_{\beta \gamma}=0, \\
& \frac{\partial f_{\beta}}{\partial \bar{\Lambda}_{\dot{\gamma}}} \mathbb{P}_{\alpha \dot{\alpha}} \delta_{\dot{\alpha} \dot{\gamma}}+\frac{\partial f_{\alpha}}{\partial \bar{\Lambda}_{\dot{\gamma}}} \mathbb{P}_{\beta \dot{\beta}} \delta_{\dot{\beta} \dot{\gamma}}=0, \\
& \bar{f}_{\beta} \bar{\Lambda}_{\gamma} \epsilon_{\alpha \gamma}+\bar{f}_{\alpha} \bar{\Lambda}_{\gamma} \epsilon_{\beta \gamma}=0, \\
& \bar{f}_{\beta} \overline{\mathbb{P}}_{\alpha \dot{\alpha}} \bar{\Lambda}_{\dot{\alpha}}+\bar{f}_{\alpha} \overline{\mathbb{P}}_{\beta \dot{\beta}} \bar{\Lambda}_{\dot{\beta}}=0 .
\end{aligned}
$$


These relations are obtained by comparing the coefficients of $\mathbb{P}_{\alpha \dot{\alpha}}, \overline{\mathbb{P}}_{\alpha \dot{\alpha}}$ and $P^{ \pm}$on both sides of eqns.(3.36) and (3.37). Similar equations are satisfied by their dotted counterparts $f_{\dot{\alpha}}$ and $\bar{f}_{\dot{\alpha}}$. There are additional conditions coming from the set of algebra in eqns.(3.38)-(3.41). The relation in eqn.(3.38) leads to the following equations involving $f_{\alpha}$ and $f_{\dot{\beta}}$ :

$$
\begin{aligned}
\frac{\partial f_{\dot{\beta}}}{\partial \bar{\Lambda}_{\gamma}} \epsilon_{\alpha \gamma} & =0, \\
\frac{\partial f_{\alpha}}{\partial \bar{\Lambda}_{\dot{\gamma}}} \epsilon_{\dot{\beta} \dot{\gamma}} & =0, \\
\frac{\partial f_{\dot{\beta}}}{\partial \bar{\Lambda}_{\dot{\gamma}}} \mathbb{P}_{\alpha \dot{\alpha}} \delta_{\dot{\alpha} \dot{\gamma}}+\frac{\partial f_{\alpha}}{\partial \bar{\Lambda}_{\gamma}} \mathbb{P}_{\dot{\beta} \beta} \delta_{\beta \gamma} & =0 .
\end{aligned}
$$

Similarly, the relation in eqn.(3.39) gives the following set of equations for the unknowns $f_{\alpha}$ and $\bar{f}_{\dot{\beta}}$ :

$$
\begin{aligned}
\frac{\partial \bar{f}_{\dot{\beta}}}{\partial \bar{\Lambda}_{\gamma}} \epsilon_{\alpha \gamma}+\frac{1}{\alpha} f_{\alpha}(\epsilon \bar{\Lambda})_{\dot{\beta}} & =0 \\
\frac{\partial \bar{f}_{\dot{\beta}}}{\partial \bar{\Lambda}_{\dot{\gamma}}} \mathbb{P}_{\alpha \dot{\alpha}} \delta_{\dot{\alpha} \dot{\gamma}}+\frac{1}{\alpha} f_{\alpha} \overline{\mathbb{P}}_{\dot{\beta} \beta} \bar{\Lambda}_{\beta} & =0 .
\end{aligned}
$$

The set of algebra in eqns.(3.40) result in the following consistency conditions for $f_{\dot{\beta}}, \bar{f}_{\alpha}$ :

$$
\begin{aligned}
\frac{\partial \bar{f}_{\alpha}}{\partial \bar{\Lambda}_{\dot{\gamma}}} \epsilon_{\dot{\beta} \dot{\gamma}}+\frac{1}{\alpha} f_{\dot{\beta}}(\epsilon \bar{\Lambda})_{\alpha} & =0, \\
\frac{\partial \bar{f}_{\alpha}}{\partial \bar{\Lambda}_{\gamma}} \mathbb{P}_{\dot{\beta} \beta} \delta_{\beta \gamma}+\frac{1}{\alpha} f_{\dot{\beta}} \overline{\mathbb{P}}_{\alpha \dot{\alpha}} \bar{\Lambda}_{\dot{\alpha}} & =0 .
\end{aligned}
$$

and finally the relations in eqn.(3.41) lead to the following conditions on $\bar{f}_{\alpha}, \bar{f}_{\dot{\beta}}$ :

$$
\begin{aligned}
& \frac{1}{\alpha} \bar{f}_{\dot{\beta}}(\epsilon \bar{\Lambda})_{\alpha}=0, \\
& \frac{1}{\alpha} \bar{f}_{\alpha}(\epsilon \bar{\Lambda})_{\dot{\beta}}=0, \\
& \bar{f}_{\dot{\beta}} \overline{\mathbb{P}}_{\alpha \dot{\alpha}} \bar{\Lambda}_{\dot{\alpha}}+\bar{f}_{\alpha} \overline{\mathbb{P}}_{\dot{\beta} \beta} \bar{\Lambda}_{\beta}=0,
\end{aligned}
$$

It is worth noting that some care has to be taken while solving the above consistency conditions, as they involve grassmann functions. We find that the set of equations (3.65)-(3.75), are solved by the functions:

$$
\begin{aligned}
f_{\alpha} & =-2 \bar{\Lambda}_{\alpha} \\
f_{\dot{\alpha}} & =2 \bar{\Lambda}_{\dot{\alpha}} \\
\bar{f}_{\alpha} & =\frac{1}{\alpha} \epsilon_{\alpha \beta} \quad \bar{\Lambda}_{\beta} \epsilon_{\dot{\gamma} \dot{\delta}} \bar{\Lambda}_{\dot{\gamma}} \bar{\Lambda}_{\dot{\delta}} \\
\bar{f}_{\dot{\alpha}} & =-\frac{1}{\alpha} \epsilon_{\dot{\alpha} \dot{\beta}} \bar{\Lambda}_{\dot{\beta}} \epsilon_{\gamma \delta} \bar{\Lambda}_{\gamma} \bar{\Lambda}_{\delta} .
\end{aligned}
$$


The solutions in eqns.(3.79)-(3.82) complete the determination of prefactors appearing in the ansatz (3.29)-(3.32) for the dynamical supercharges. We now go on to determine the prefactors of the interaction hamiltonian and hence, the cubic open-string vertex operator. Substituting the ansatz given in eqn.(3.28) for the interaction hamiltonian in eqn.(3.35), we end up with the following relations:

$$
\begin{aligned}
\frac{\partial \bar{f}_{\beta}}{\partial \bar{\Lambda}_{\gamma}} \epsilon_{\alpha \gamma} & =2 \delta_{\alpha \beta} f^{-} \\
\frac{1}{\alpha} f_{\alpha} \epsilon_{\beta \gamma} \bar{\Lambda}_{\gamma} & =2 \delta_{\alpha \beta} f^{+} \\
\frac{\partial \bar{f}_{\beta}}{\partial \bar{\Lambda}_{\dot{\gamma}}} \mathbb{P}_{\alpha \dot{\alpha}} \delta_{\dot{\alpha} \dot{\gamma}}+\frac{1}{\alpha} f_{\alpha} \overline{\mathbb{P}}_{\beta \dot{\beta}} \bar{\Lambda}_{\dot{\beta}} & =2 \delta_{\alpha \beta}\left(\mathbb{P}_{\gamma \dot{\gamma}} f^{\gamma \dot{\gamma}}+\overline{\mathbb{P}}_{\gamma \dot{\gamma}} \bar{f}^{\gamma \dot{\gamma}}\right) .
\end{aligned}
$$

Now, using eqns.(3.79)-(3.82) in eqns.(3.83)-(3.85), the solutions for the unknown functions in the ansatz for the interaction hamiltonian $(3.28)$ are given as $f_{\alpha}, \bar{f}_{\alpha}$ :

$$
\begin{aligned}
f^{-} & =\frac{1}{2 \alpha} \epsilon_{\dot{\alpha} \dot{\beta}} \quad \bar{\Lambda}_{\dot{\alpha}} \bar{\Lambda}_{\dot{\beta}}, \\
f^{+} & =-\frac{1}{2 \alpha} \epsilon_{\alpha \beta} \bar{\Lambda}_{\alpha} \bar{\Lambda}_{\beta}, \\
f_{\alpha \dot{\alpha}} & =-\frac{1}{\alpha} \epsilon_{\alpha \gamma} \epsilon_{\dot{\alpha} \dot{\delta}} \bar{\Lambda}_{\gamma} \bar{\Lambda}_{\dot{\delta}} \\
\bar{f}_{\alpha \dot{\alpha}} & =-\frac{1}{\alpha} \bar{\Lambda}_{\alpha} \bar{\Lambda}_{\dot{\alpha}} .
\end{aligned}
$$

The dotted counterpart to equation (3.35) leads to slightly different conditions than in eqns. (3.83)-(3.85). However, the final answer for the prefactor of the interaction hamiltonian turns out to be identical to the ones in eqns. (3.86)-(3.89).

Hence, the solutions given in eqns.(3.79)-(3.82) and (3.86)-(3.89) determine the complete cubic interaction vertex and the supercharges for an open string on a $D 7$-brane at the level of zero modes when substituted in eqns.(3.28)-(3.32).

\section{Superstring vertex}

In this section we generalize our results of the previous section to the full string theory by following the approach of $[55,26]$ for both closed and open strings earlier. Before proceeding, we note, that at $\tau=0$ the bosonic mode expansions for Neumann directions given in eqns.(2.4) can be written in the form:

$$
X^{r}(\sigma)=x_{0}^{r}+i \sum_{n=1}^{\infty}\left[\frac{1}{\omega_{n}}\left(\alpha_{n}^{r}-\alpha_{-n}^{r}\right) \cos \frac{n \sigma}{\mid \alpha_{\mid}}\right],
$$


and their conjugate momenta are given as :

$$
P^{r}(\sigma)=\frac{1}{\pi|\alpha|}\left[p_{0}^{r}+\sum_{n=1}^{\infty}\left(\alpha_{n}^{r}+\alpha_{-n}^{r}\right) \cos \frac{n \sigma}{\mid \alpha_{\mid}}\right] .
$$

For Dirichlet directions we have:

$$
X^{r^{\prime}}(\sigma)=\sum_{n=1}^{\infty}\left[\frac{1}{\omega_{n}}\left(\alpha_{n}^{r^{\prime}}+\alpha_{-n}^{r^{\prime}}\right) \sin \frac{n \sigma}{\mid \alpha_{\mid}}\right],
$$

with their conjugate momenta being:

$$
P^{r^{\prime}}(\sigma)=\frac{1}{\pi|\alpha|}\left[-i \sum_{n=1}^{\infty}\left(\alpha_{n}^{r^{\prime}}-\alpha_{-n}^{r^{\prime}}\right) \sin \frac{n \sigma}{|\alpha|}\right] .
$$

To write down the mode expansions of fermions $S^{1}$ and $S^{2}$, one can use a decomposition similar to the one in previous section for zero modes, i.e. eqn. (3.7). One also notes using eqns. (2.7) that they satisfy $S^{1}(\sigma)=\Omega S^{2}(-\sigma)$. We therefore use the following decompositions for the fermion fields and their modes in eqn. (2.7) :

$$
S^{2}(\sigma)=\left(\begin{array}{c}
\lambda_{\dot{\alpha}}^{1}(\sigma) \\
\lambda_{\alpha}^{1}(\sigma) \\
\lambda_{\dot{\alpha}}^{2}(\sigma) \\
\lambda_{\alpha}^{2}(\sigma)
\end{array}\right), \quad S_{n}=\left(\begin{array}{c}
\lambda_{n \dot{\alpha}}^{1} \\
\lambda_{n \alpha}^{1} \\
\lambda_{n \dot{\alpha}}^{2} \\
\lambda_{n \alpha}^{2}
\end{array}\right) .
$$

The complex combinations $\lambda^{\alpha}(\sigma), \bar{\lambda}^{\alpha}(\sigma)$ etc, which can also be identified as components of $(1 \pm i \Omega) S^{2}$, then have the following mode expansions:

$$
\begin{aligned}
\lambda^{\alpha}(\sigma) & =\frac{1}{\alpha} \sum_{-\infty}^{\infty} R_{n}^{\alpha} e^{i n \sigma /|\alpha|}, & \tilde{\lambda}^{\alpha}(\sigma) & =\frac{1}{\alpha} \sum_{-\infty}^{\infty} R_{n}^{\alpha} e^{-i n \sigma /|\alpha|} \\
\lambda^{\dot{\alpha}}(\sigma) & =\frac{1}{\alpha} \sum_{-\infty}^{\infty} R_{n}^{\dot{\alpha}} e^{i n \sigma /|\alpha|}, & \tilde{\lambda}^{\dot{\alpha}}(\sigma) & =\frac{1}{\alpha} \sum_{-\infty}^{\infty} R_{n}^{\dot{\alpha}} e^{-i n \sigma /|\alpha|} \\
\bar{\lambda}^{\alpha}(\sigma) & =\frac{1}{|\alpha|} \sum_{-\infty}^{\infty} \bar{R}_{n}^{\alpha} e^{i n \sigma /|\alpha|}, & \tilde{\bar{\lambda}}^{\alpha}(\sigma) & =\frac{1}{|\alpha|} \sum_{-\infty}^{\infty} \bar{R}_{n}^{\alpha} e^{-i n \sigma /|\alpha|} \\
\bar{\lambda}^{\dot{\alpha}}(\sigma) & =\frac{1}{|\alpha|} \sum_{-\infty}^{\infty} \bar{R}_{n}^{\dot{\alpha}} e^{i n \sigma /|\alpha|}, & \tilde{\bar{\lambda}}^{\dot{\alpha}}(\sigma) & =\frac{1}{|\alpha|} \sum_{-\infty}^{\infty} \bar{R}_{n}^{\dot{\alpha}} e^{-i n \sigma /|\alpha|}
\end{aligned}
$$

In writing the above form of the mode expansions, we have used the explicit representation of $\Omega$ and $\Pi$ matrices given in eqn.(3.14), with $R_{n}$ 's defined in terms of fermionic creation and annihilation operators as:

$$
\begin{aligned}
R_{n}^{\alpha} & =i \sqrt{\alpha} c_{n}\left(\lambda_{n}^{\alpha}+\rho_{n} \lambda_{-n}^{\alpha}\right), \\
R_{n}^{\dot{\alpha}} & =i \sqrt{\alpha} c_{n}\left(\lambda_{n}^{\dot{\alpha}}-\rho_{n} \lambda_{-n}^{\dot{\alpha}}\right), \\
\bar{R}_{n}^{\alpha} & =-i \sqrt{\alpha} c_{n}\left(\bar{\lambda}_{n}^{\alpha}-\rho_{n} \bar{\lambda}_{-n}^{\alpha}\right), \\
\bar{R}_{n}^{\dot{\alpha}} & =-i \sqrt{\alpha} c_{n}\left(\bar{\lambda}_{n}^{\dot{\alpha}}+\rho_{n} \bar{\lambda}_{-n}^{\dot{\alpha}}\right),
\end{aligned}
$$


and $\lambda_{n}^{\alpha, \dot{\alpha}}=\left(\lambda_{1 n}-i \lambda_{2 n}\right)^{\alpha, \dot{\alpha}}$ and $\bar{\lambda}_{n}^{\alpha, \dot{\alpha}}=\left(\lambda_{1 n}+i \lambda_{2 n}\right)^{\alpha, \dot{\alpha}}$. We also have $R_{0}^{\alpha}=\sqrt{\alpha} \lambda^{\alpha}, R_{0}^{\dot{\alpha}}=$ $\sqrt{\alpha} \lambda^{\dot{\alpha}}, \bar{R}_{0}^{\alpha}=\sqrt{\alpha} \bar{\lambda}^{\alpha}$ and $\bar{R}_{0}^{\dot{\alpha}}=\sqrt{\alpha} \bar{\lambda}^{\dot{\alpha}}$ for the zero modes. One can find the commutation relations of $R^{\prime}$ s, using the ones for $\lambda^{\prime}$ s given in eqns. (2.12) and (2.16):

$$
\begin{aligned}
& \left\{R_{m}^{\alpha}, \bar{R}_{n}^{\beta}\right\}=\alpha \delta_{m+n, 0} \delta^{\alpha \beta} \\
& \left\{R_{m}^{\alpha}, R_{n}^{\beta}\right\}=\left\{\bar{R}_{m}^{\alpha}, \bar{R}_{n}^{\beta}\right\}=0
\end{aligned}
$$

with similar commutation relations for the $R^{\dot{\alpha}}$ 's as well. The bosonic and fermionic mode expansions given in eqns.(4.1), (4.2) and (4.6)-(4.9), have been written in a form so as to match with the ones given in [55]. With this matching, it is possible to determine the exlicit form of the kinematical part of the open string vertex from the Fourier modes of the constraints:

$$
\begin{aligned}
& \sum_{r=1}^{3} \varepsilon_{r} x_{r}\left(\sigma_{r}\right)\left|E_{b}\right\rangle=0, \quad \sum_{r=1}^{3} p_{r}\left(\sigma_{r}\right)\left|E_{b}\right\rangle=0, \\
& \sum_{r=1}^{3} \varepsilon_{r} \lambda_{(r) \alpha, \dot{\alpha}}\left(\sigma_{r}\right)\left|E_{f}\right\rangle=0, \quad \sum_{r=1}^{3} \bar{\lambda}_{(r) \alpha, \dot{\alpha}}\left(\sigma_{r}\right)\left|E_{f}\right\rangle=0 .
\end{aligned}
$$

Here, $r$ is the string index and $\varepsilon_{r}$ is +1 for an incoming string and -1 for an outgoing one. These conditions generalize the constraints given for the zero mode vertex in eqns.(3.17)-(3.19), to the full open superstring. This part of the vertex has been derived in sufficient detail in [26] with further corrections given in [30], for the case of closed strings in PP-wave background.

To determine the bosonic part of the kinematical vertex for our case, we write down the constraints given in eqn.(4.16), in terms of their Fourier modes as:

$$
\sum_{r} \sum_{-\infty}^{\infty} \alpha_{r} X_{m n}^{(r)} x_{n(r)}\left|E_{b}\right\rangle=0, \quad \sum_{r} \sum_{-\infty}^{\infty} X_{m n}^{(r)} p_{n(r)}\left|E_{b}\right\rangle=0
$$

where the matrix $X_{m n}^{(r)}$, as well as other functions appearing below, are defined in the appendix. These constraints on the vertex turn out to be slightly different for the Neumann and Dirichlet directions. For Neumann directions we have $(m>0)$ :

$$
\begin{aligned}
{\left[\sum_{r=1}^{3} \sum_{n=1}^{\infty} \alpha_{r} X^{(r)} C^{-1 / 2} C_{(r)}^{-1 / 2}\left(a_{n}^{(r)}-a_{-n}^{(r)}\right)+i \alpha B \mathbb{R}\right]\left|E_{b}\right\rangle } & =0 \\
{\left[\sum_{r=1}^{3} \sum_{n=1}^{\infty} X^{(r)} C^{-1 / 2} C_{(r)}^{1 / 2}\left(a_{n}^{(r)}+a_{-n}^{(r)}\right)+B \mathbb{P}\right]\left|E_{b}\right\rangle } & =0
\end{aligned}
$$

and those in the Dirichlet directions are:

$$
\begin{gathered}
{\left[\sum_{r=1}^{3} \sum_{n=1}^{\infty} X^{(r)} C^{1 / 2} C_{(r)}^{-1 / 2}\left(a_{n}^{(r)}+a_{-n}^{(r)}\right)\right]\left|E_{b}\right\rangle=0} \\
{\left[\sum_{r=1}^{3} \sum_{n=1}^{\infty} \frac{1}{\alpha_{r}} X^{(r)} C^{1 / 2} C_{(r)}^{1 / 2}\left(a_{n}^{(r)}-a_{-n}^{(r)}\right)\right]\left|E_{b}\right\rangle=0 .}
\end{gathered}
$$


Here $a_{n}^{\dagger}=\frac{\alpha_{n}^{\dagger}}{\sqrt{\omega_{n}}}$ and $a_{n}=\frac{\alpha_{n}}{\sqrt{\omega_{n}}}$, satisfy $\left[a_{n}, a_{m}^{\dagger}\right]=\delta_{m n}$. Hence, the bosonic part of the kinematical vertex satisfying the coordinate continuity and momentum conservation constraints given in terms of their Fourier modes in eqns.(4.19)-(4.22), is given as [26, 31]:

$$
\left|E_{b}\right\rangle \sim \exp \left\{\frac{1}{2} \sum_{r, s=1}^{3} \sum_{m, n} a_{m(r)}^{\dagger} \bar{N}_{m n}^{r s} a_{n(s)}^{\dagger}\right\}|0\rangle_{123}
$$

where $|0\rangle_{123}=|0\rangle_{1} \otimes|0\rangle_{2} \otimes|0\rangle_{3}$ is annihilated by $a_{n}$. In the above equation, $\bar{N}_{m n}^{r s}$ are the bosonic Neumann matrices defined in the appendix and the summation is over appropriate modes of Neumann and Dirichlet directions[58].

Similarly, the fermionic part of the kinematical vertex can be determined by writing down the constraints given in eqn.(4.17) in terms of their Fourier modes. The cosine and sine Fourier modes of the $\lambda^{\alpha}$ conditions are:

$$
\begin{aligned}
{\left[\sum_{r=1}^{3} \sum_{n=1}^{\infty} X^{(r)} C^{-1 / 2}\left(R_{n}^{\alpha(r)}+R_{-n}^{\alpha(r)}\right)-\sqrt{2} \alpha B \Theta^{\alpha}\right]\left|E_{f}\right\rangle } & =0 \\
{\left[\sum_{r=1}^{3} \sum_{n=1}^{\infty} \frac{1}{\alpha_{r}} X^{(r)} C^{1 / 2}\left(R_{n}^{\alpha(r)}-R_{-n}^{\alpha(r)}\right)\right]\left|E_{f}\right\rangle } & =0
\end{aligned}
$$

and the ones for $\bar{\lambda}^{\alpha}$ conditions are:

$$
\begin{aligned}
{\left[\sum_{r=1}^{3} \sum_{n=1}^{\infty} X^{(r)} C^{-1 / 2}\left(\bar{R}_{n}^{\alpha(r)}+\bar{R}_{-n}^{\alpha(r)}\right)+\frac{1}{\sqrt{2}} B \bar{\Lambda}^{\alpha}\right]\left|E_{f}\right\rangle } & =0 \\
{\left[\sum_{r=1}^{3} \sum_{n=1}^{\infty} \frac{1}{\alpha_{r}} X^{(r)} C^{1 / 2}\left(\bar{R}_{n}^{\alpha(r)}-\bar{R}_{-n}^{\alpha(r)}\right)\right]\left|E_{f}\right\rangle } & =0
\end{aligned}
$$

There will be similar conditions for the Fourier modes of $\lambda^{\dot{\alpha}}$ and $\bar{\lambda}^{\dot{\alpha}}$ as well.

Hence, the fermionic part of the kinematical vertex satisfying the constraints given in terms of their Fourier modes in eqns.(4.24)-(4.27) (and their dotted counterparts), turns out to be [55, $26,31]$ :

$$
\begin{aligned}
\left|E_{f}\right\rangle \sim \exp \left[\sum_{r, s=1}^{3} \sum_{m, n=1}^{\infty}\left(\lambda_{-m(r)}^{\alpha} Q_{m n \alpha \beta}^{r s} \bar{\lambda}_{-n(s)}^{\beta}+\lambda_{-m(r)}^{\dot{\alpha}} Q_{m n \dot{\alpha} \dot{\beta}}^{r s} \bar{\lambda}_{-n(s)}^{\dot{\alpha}}\right)\right. \\
\left.-\sum_{r=1}^{3} \sum_{m=1}^{\infty}\left(\lambda_{-m(r)}^{\alpha} Q_{m \alpha \beta}^{r} \bar{\Theta}^{\alpha}+\lambda_{-m(r)}^{\dot{\alpha}} Q_{m \dot{\alpha} \dot{\beta}}^{r} \bar{\Theta}^{\dot{\alpha}}\right)\right]\left|E_{f}^{0}\right\rangle,
\end{aligned}
$$

where $\bar{\Theta} \equiv \frac{1}{\alpha_{3}}\left(\bar{\lambda}_{0(1)}-\bar{\lambda}_{0(2)}\right)$ and $\left|E_{f}^{0}\right\rangle$ is the zero-mode part of the fermionic vertex given in eqn.(3.22). The matrices $Q_{m n}^{r s}$ and $Q_{m}^{r}$ are the fermionic Neumann matrices. Their relation to the bosonic Neumann matrices are given in the appendix.

One can also see the structure of the fermionic part of the kinematic vertex written above in eqn.(4.28), by combining the mode expansions given in eqns.(4.6)-(4.9) and (4.10)-(4.13), and comparing with the ones in eqns. (2.6) and (2.7) of [30]. Alternatively, for example, the 
mode expansion for the quantity $(1-i \Omega) S_{2}$ (as given in component forms in (4.6)-(4.9)) can be written as:

$$
(1-i \Omega) S_{2}(\sigma)=(1-i \Omega)\left[S_{0}+i \sum_{n=1}\left\{\theta_{n} \cos \frac{n \sigma}{|\alpha|}+i \theta_{-n} \sin \frac{n \sigma}{|\alpha|}\right\}\right]
$$

where,

$$
\theta_{n}=i \sqrt{\alpha} c_{n}\left[\left(1-\rho_{n} \Pi\right) S_{n}+\left(1+\rho_{n} \Pi\right) S_{-n}\right]
$$

Since these expansions are identical to the ones given in [30], our Fermionic Neumann matrices are also identical. In appendix we write their expressions in component forms as well. We also note that due to a specific choice of basis, matrix $\Omega$ does not appear explicitly in our modes $\theta_{n}$, as well as in kinematic vertex.

Having discussed the kinematical part of the vertex $\left|V_{k i n}\right\rangle$, we go on to determine the prefactors, that multiply the vertex and the dynamical generators. First, we form the following combination of dynamical supercharges given in eqn.(2.29):

$$
\begin{array}{r}
\sqrt{2 p^{+}} Q^{-}=\frac{1}{2 \pi \alpha^{\prime} p^{+}} \int_{0}^{2 \pi \alpha^{\prime}\left|p^{+}\right|} d \sigma\left[\left\{\partial_{\tau} X^{r} \gamma^{r}+\partial_{\sigma} X^{r^{\prime}} \gamma^{r^{\prime}}+m X_{r} \gamma^{r} \Omega \Pi\right\}\left(S^{2}+\Omega^{T} S^{1}\right)\right. \\
\left.+\left\{\partial_{\tau} X^{r^{\prime}} \gamma^{r^{\prime}}+\partial_{\sigma} X^{r} \gamma^{r}-m X_{r^{\prime}} \gamma^{r^{\prime}} \Omega \Pi\right\}\left(S^{2}-\Omega^{T} S^{1}\right)\right] .
\end{array}
$$

Similar combinations of supercharges have been considered in [53], for the case of open strings in $D=10$ which in the present context will correspond to a $D 9$-brane in flat space. Using the form of matrices $\Omega$ and $\Pi$ given in eqn.(3.14), we can now write down the various components of dynamical supercharges given in eqn.(4.31) as:

$$
\begin{aligned}
Q_{\alpha}^{-}=\int d \sigma & \frac{1}{\alpha}\left[\left\{P_{\alpha \dot{\alpha}} \lambda_{\dot{\alpha}}^{+}+P^{+}\left(\epsilon \lambda^{+}\right)_{\alpha}+\partial_{\sigma} \tilde{X}^{-} \bar{\lambda}_{\alpha}^{+}-i m\left(X_{\alpha \dot{\alpha}} \lambda_{\dot{\alpha}}^{+}-X^{+}\left(\epsilon \lambda^{+}\right)_{\alpha}\right)\right\}\right. \\
+ & \left.\left\{\tilde{P}^{-}\left(\bar{\lambda}^{-}\right)_{\alpha}+\partial_{\sigma} X_{\alpha \dot{\alpha}} \lambda_{\dot{\alpha}}^{-}+\partial_{\sigma} X^{+}\left(\epsilon \lambda^{-}\right)_{\alpha}-i m \tilde{X}^{-} \bar{\lambda}_{\alpha}^{-}\right\}\right], \\
\bar{Q}_{\alpha}^{-}=\int d \sigma & \frac{1}{\alpha}\left[\left\{\bar{P}_{\alpha \dot{\alpha}} \bar{\lambda}_{\dot{\alpha}}^{+}+P^{-}\left(\epsilon \bar{\lambda}^{+}\right)_{\alpha}+\partial_{\sigma} \tilde{X}^{+} \lambda_{\alpha}^{+}+i m\left(\bar{X}_{\alpha \dot{\alpha}} \bar{\lambda}_{\dot{\alpha}}^{+}-X^{-}\left(\epsilon \bar{\lambda}^{+}\right)_{\alpha}\right)\right\}\right. \\
+ & \left.\left\{\tilde{P}^{+}\left(\lambda^{-}\right)_{\alpha}+\partial_{\sigma} \bar{X}_{\alpha \dot{\alpha}} \bar{\lambda}_{\dot{\alpha}}^{-}+\partial_{\sigma} X^{-}\left(\epsilon \bar{\lambda}^{-}\right)_{\alpha}+i m \tilde{X}^{+} \lambda_{\alpha}^{-}\right\}\right], \\
Q_{\dot{\alpha}}^{-}=\int d \sigma & \frac{1}{\alpha}\left[\left\{P_{\dot{\alpha} \alpha} \lambda_{\alpha}^{+}+P^{-}\left(\epsilon \lambda^{+}\right)_{\dot{\alpha}}-\partial_{\sigma} \tilde{X}^{-} \bar{\lambda}_{\dot{\alpha}}^{+}+i m\left(X_{\dot{\alpha} \alpha} \lambda_{\alpha}^{+}-X^{-}\left(\epsilon \lambda^{+}\right)_{\dot{\alpha}}\right)\right\}\right. \\
+ & \left.\left\{-\tilde{P}^{+}\left(\bar{\lambda}^{-}\right)_{\dot{\alpha}}+\partial_{\sigma} X_{\dot{\alpha} \alpha} \lambda_{\alpha}^{-}+\partial_{\sigma} X^{+}\left(\epsilon \lambda^{-}\right)_{\dot{\alpha}}-i m \tilde{X}^{-} \bar{\lambda}_{\dot{\alpha}}^{-}\right\}\right], \\
\bar{Q}_{\dot{\alpha}}^{-}=\int d \sigma & \frac{1}{\alpha}\left[\left\{\bar{P}_{\dot{\alpha} \alpha} \bar{\lambda}_{\alpha}^{+}+P^{+}\left(\epsilon \bar{\lambda}^{+}\right)_{\dot{\alpha}}-\partial_{\sigma} \tilde{X}^{+} \lambda_{\dot{\alpha}}^{+}-i m\left(\bar{X}_{\dot{\alpha} \alpha} \bar{\lambda}_{\alpha}^{+}-X^{+}\left(\epsilon \bar{\lambda}^{+}\right)_{\dot{\alpha}}\right)\right\}\right. \\
+ & \left.\left\{-\tilde{P}^{-}\left(\lambda^{-}\right)_{\dot{\alpha}}+\partial_{\sigma} \bar{X}_{\dot{\alpha} \alpha} \bar{\lambda}_{\alpha}^{-}+\partial_{\sigma} X^{-}\left(\epsilon \bar{\lambda}^{-}\right)_{\dot{\alpha}}+i m \tilde{X}^{+} \lambda_{\dot{\alpha}}^{-}\right\}\right],
\end{aligned}
$$

with the definitions $\lambda^{ \pm} \equiv \lambda(\sigma) \pm \tilde{\lambda}(\sigma)=\lambda(\sigma) \pm \lambda(-\sigma)$. 
For stringy generalization of operators $\mathbb{P}$ and $\Lambda$ appearing in the last section one needs to take care of the singularity at $\sigma=\pi \alpha_{1}$ by defining $[55,26]$ :

$$
\begin{aligned}
P\left|V_{k i n}\right\rangle & =\lim _{\sigma \rightarrow \pi \alpha_{(1)}}-2 \pi \sqrt{-\alpha}\left(\pi \alpha_{(1)}-\sigma\right)^{1 / 2}\left(P_{(1)}(\sigma)+P_{(1)}(-\sigma)\right)\left|V_{k i n}\right\rangle, \\
\partial X\left|V_{k i n}\right\rangle & =\lim _{\sigma \rightarrow \pi \alpha_{(1)}}-2 \pi \sqrt{-\alpha}\left(\pi \alpha_{(1)}-\sigma\right)^{1 / 2}\left(\partial_{\sigma} X_{(1)}(\sigma)+\partial_{\sigma} X_{(1)}(-\sigma)\right)\left|V_{k i n}\right\rangle, \\
\bar{\Lambda}\left|V_{k i n}\right\rangle & =\lim _{\sigma \rightarrow \pi \alpha_{(1)}}-2 \pi \sqrt{-\alpha}\left(\pi \alpha_{(1)}-\sigma\right)^{1 / 2}\left(\bar{\lambda}_{(1)}(\sigma)+\bar{\lambda}_{(1)}(-\sigma)\right)\left|V_{k i n}\right\rangle .
\end{aligned}
$$

with similar definitions for $\bar{P}, \bar{X}, X^{ \pm}, \tilde{X}^{ \pm}$. Also, the same thing holds for the second and the third strings. The commutation relations involving $Q_{(r) \alpha}^{-}{ }^{\prime} s$ then have identical forms as given in (3.53)-(3.60). However, in the present case, there are additional relations which are non-zero and useful in determining the prefactors:

$$
\begin{aligned}
\sum_{r=1}^{3}\left\{Q_{(r) \alpha}^{-}, \Lambda_{\beta}\right\} & =\partial_{\sigma} \tilde{X}^{-} \delta_{\alpha \beta}, \\
\sum_{r=1}^{3}\left\{\bar{Q}_{(r) \alpha}^{-}, \bar{\Lambda}_{\beta}\right\} & =\partial_{\sigma} \tilde{X}^{+} \delta_{\alpha \beta},
\end{aligned}
$$

Similarly, for the $Q_{(r) \dot{\alpha}}^{-}$'s, we have:

$$
\begin{aligned}
\sum_{r=1}^{3}\left\{Q_{(r) \dot{\alpha}}^{-}, \Lambda_{\dot{\beta}}\right\} & =-\partial_{\sigma} \tilde{X}^{-} \delta_{\dot{\alpha} \dot{\beta}}, \\
\sum_{r=1}^{3}\left\{\bar{Q}_{(r) \dot{\alpha}}^{-}, \bar{\Lambda}_{\dot{\beta}}\right\} & =-\partial_{\sigma} \tilde{X}^{+} \delta_{\dot{\alpha} \dot{\beta}} .
\end{aligned}
$$

Using $X\left|V_{\text {kin }}\right\rangle=0$ and $\Lambda\left|V_{\text {kin }}\right\rangle=0$ as in [26], the state $\left|V_{\text {kin }}\right\rangle$ can be shown to satisfy:

$$
\begin{aligned}
\sum_{r=1}^{3} Q_{(r) \alpha}^{-}\left|V_{k i n}\right\rangle & =-\frac{1}{\alpha} \partial_{\sigma} \tilde{X}^{-} \bar{\Lambda}_{\alpha}\left|V_{k i n}\right\rangle, \\
\sum_{r=1}^{3} Q_{(r) \dot{\alpha}}^{-}\left|V_{k i n}\right\rangle & =\frac{1}{\alpha} \partial_{\sigma} \tilde{X}^{-} \bar{\Lambda}_{\dot{\alpha}}\left|V_{k i n}\right\rangle, \\
\sum_{r=1}^{3} \bar{Q}_{(r) \alpha}^{-}\left|V_{k i n}\right\rangle & =-\frac{1}{\alpha}\left[P_{\alpha \dot{\alpha}} \bar{\Lambda}_{\dot{\alpha}}+P^{-}(\epsilon \bar{\Lambda})_{\alpha}\right]\left|V_{k i n}\right\rangle, \\
\sum_{r=1}^{3} \bar{Q}_{(r) \dot{\alpha}}^{-}\left|V_{k i n}\right\rangle & =-\frac{1}{\alpha}\left[P_{\dot{\alpha} \alpha} \bar{\Lambda}_{\alpha}+P^{+}(\epsilon \bar{\Lambda})_{\dot{\alpha}}\right]\left|V_{k i n}\right\rangle .
\end{aligned}
$$

In writing the action of the dynamical supercharges on the vertex, we have also made use of the fact that $\partial_{\sigma} X^{r}\left|V_{k i n}\right\rangle=0=P^{r^{\prime}}\left|V_{k i n}\right\rangle$, where $r$ and $r^{\prime}$ are Neumann and Dirichlet directions respectively. These relations can be explicitly verifed in the oscillator basis representation $[27,30,31]$, keeping in mind that the mode expansions for string coordinates in the Neumann directions are given in terms of cosines and for Dirichlet directions in terms of sines. 
Now, we can repeat the exercise of section-3 to determine the prefactors for the superstring vertex, by demanding that the PP-wave superalgebra in eqns.(3.35)-(3.38) is satisfied. For $Q_{a}$ 's the form of ansatz is same as given in eqns.(3.29)-(3.32) and for the interaction Hamiltonian we have,

$$
\begin{aligned}
|H\rangle= & {\left[P_{\alpha \dot{\alpha}} f^{\alpha \dot{\alpha}}+\bar{P}_{\alpha \dot{\alpha}} \bar{f}^{\alpha \dot{\alpha}}+P^{+} f^{-}\right.} \\
& \left.+P^{-} f^{+}+\partial_{\sigma} \tilde{X}^{-} \tilde{f}^{+}+\partial_{\sigma} \tilde{X}^{+} \tilde{f}^{-}\right]\left|V_{k i n}\right\rangle
\end{aligned}
$$

wherein, the quantities $f$ 's are again the functions of $\bar{\Lambda}_{\alpha}, \bar{\Lambda}_{\dot{\alpha}}$ which are generalized to include all the string non-zero modes, with the definitions as in eqn.(4.36). Similarly, $P^{ \pm}, P_{\alpha \dot{\alpha}}$ etc. are stringy generalizations of $\mathbb{P}^{\prime}$ 's appearing in section-3, and contain all the non-zero creation operators. These quantities can be explicitly realized in the oscillator basis as well. We also note that, unlike in flat space, there may be some $\mu$ dependent normalizations in the above ansatz for the interaction vertex, corresponding to the difference in functional and oscillator basis expressions [30]. However, in the following analysis, we ignore these normalizations.

Using the supercharges given in eqns.(4.32)-(4.35) and the relations (4.37)-(4.44) as well as (3.45)-(3.60) in the PP-wave superalgebra in eqns.(3.35)-(3.38), we get several consistency conditions for the unknown functions $f$ appearing in eqn.(4.45). Most of these conditions are identical to those given in eqns.(3.65)-(3.75). However, the functions $f$ need to satisfy certain additional conditions. First, the relations given in eqns.(3.36) and (3.37) imply the following conditions for $f_{\alpha}$ and $\bar{f}_{\alpha}$ :

$$
\begin{aligned}
f_{\beta} \bar{\Lambda}_{\alpha}+f_{\alpha} \bar{\Lambda}_{\beta} & =0 \\
\frac{\partial \bar{f}_{\beta}}{\partial \bar{\Lambda}_{\gamma}} \delta_{\alpha \gamma}+\frac{\partial \bar{f}_{\alpha}}{\partial \bar{\Lambda}_{\gamma}} \delta_{\beta \gamma} & =0 .
\end{aligned}
$$

Equations (3.38) and (3.39) lead to further restrictions on $f_{\dot{\beta}}, f_{\alpha}$, and $\bar{f}_{\dot{\beta}}$ as given below:

$$
\begin{aligned}
f_{\dot{\beta}} \bar{\Lambda}_{\alpha}+f_{\alpha} \bar{\Lambda}_{\dot{\beta}} & =0, \\
\bar{f}_{\dot{\beta}} \bar{\Lambda}_{\alpha} & =0, \\
\frac{\partial f_{\alpha}}{\partial \bar{\Lambda}_{\dot{\gamma}}} \delta_{\dot{\beta} \dot{\gamma}} & =0 .
\end{aligned}
$$

Equation (3.40) gives the following consistency conditions for $f_{\dot{\beta}}$ and $\bar{f}_{\alpha}$ :

$$
\frac{\partial f_{\dot{\beta}}}{\partial \bar{\Lambda}_{\gamma}} \delta_{\alpha \gamma}=0, \quad \bar{f}_{\alpha} \bar{\Lambda}_{\dot{\beta}}=0
$$

Finally, eqn.(3.41) results in:

$$
\frac{\partial \bar{f}_{\dot{\beta}}}{\partial \bar{\Lambda}_{\gamma}} \delta_{\alpha \gamma}-\frac{\partial \bar{f}_{\alpha}}{\partial \bar{\Lambda}_{\dot{\gamma}}} \delta_{\dot{\beta} \dot{\gamma}}=0
$$


We note that the solutions given in eqns.(3.79)-(3.82), also satisfy the additional conditions given in the above equations (4.46)-(4.52) for the superstring case when proper stringy generalizations of various operators are used. The unknown functions $\tilde{f}^{+}$and $\tilde{f}^{-}$, appearing in the ansatz for the interaction Hamiltonian given in eqn.(4.45) can now be determined by using the algebra in eqn.(3.35), which leads to the following equations:

$$
2 \tilde{f}^{+} \delta_{\alpha \beta}+\frac{1}{\alpha} \bar{f}_{\beta} \bar{\Lambda}_{\alpha}=0
$$

and,

$$
2 \tilde{f}^{-} \delta_{\alpha \beta}-\frac{\partial f_{\alpha}}{\partial \bar{\Lambda}_{\gamma}} \delta_{\beta \gamma}=0 .
$$

Using the solutions for $\bar{f}_{\beta}$ and $f_{\alpha}$ given in equations (3.79)-(3.82), in the above equation, we get (after taking into account the stringy generalization for $\Lambda, \bar{\Lambda}$ etc. given in eqn.(4.36)):

$$
\tilde{f}^{-}=-1, \quad \text { and } \quad \tilde{f}^{+}=\frac{1}{4 \alpha^{2}} \epsilon_{\alpha \beta} \quad \bar{\Lambda}_{\alpha} \bar{\Lambda}_{\beta} \quad \epsilon_{\dot{\alpha} \dot{\beta}} \bar{\Lambda}_{\dot{\alpha}} \bar{\Lambda}_{\dot{\beta}}
$$

The solutions given in equations (3.79)-(3.82), (3.86)-(3.89) and (4.55) then determine the prefactors, and hence the complete cubic superstring vertex and dynamical supercharges for an open string ending on a D7-brane.

\section{Conclusions}

In this paper, we have discussed the construction of cubic interaction vertex for an open string ending on a D7-brane in PP-wave background, using Green-Schwarz light-cone string field theory formalism. We explicitly determined the prefactors for the cubic interaction vertex and the dynamical supercharges at the level of zero modes and then generalized it to include all the stringy excitations. This was achieved by writing down all the symmetry generators in terms of the $D 7$-brane symmetry structure $S U(2)_{L} \times S U(2)_{R} \times S O(2)_{1} \times S O(2)_{2}$. It is interesting to note that prefactors for the interaction vertex resemble those of the flat space [55]. It will be nice to give an oscillator basis realization of the prefactors appearing in the superstring vertex as given for the flat space case in [55]. Such expression for the closed strings in PP-wave background have already been given in [27, 30, 31].

The proposal of [9] for closed strings, amounts to calculating cubic string interaction amplitudes in the light-cone string field approach, and relating them to the three-point correlation functions on the super Yang-Mills side. In the present case, having found the cubic open string interaction vertex, it would be interesting to calculate the dual field theory correlation functions and three-point functions using the determinant operators given by [49], or in terms of defect CFT correlation functions. This would provide a check of the BMN duality, for open strings even at the level of interactions. It would also be worthwhile to address the question 
of the existence of higher order open string interactions in PP-wave background, as has been discussed by [19] for the case of closed strings.

Finally, although our construction of the cubic vertex was for an open string on a $D 7$-brane in PP-wave background, similar analysis should hold for the case of open string interactions on other D3 and D5 branes as well. We hope to get back to these issues in future.

\section{Acknowledgments}

We are thankful to Ari Pankiewicz for pointing out to us the relationship between functional and oscillator basis expressions and for various coments on the draft. We would also like to thank Sanjay for useful discussions.

\section{A Matrices appearing in the kinematic vertex}

1. The Matrix $M^{r s}$ appearing in the bosonic kinematic vertex in eqn.(3.21) is given in [26]:

$$
M^{r s}=\left(\begin{array}{ccc}
\beta+1 & -\sqrt{-\beta(1+\beta)} & -\sqrt{-\beta} \\
-\sqrt{-\beta(1+\beta)} & -\beta & -\sqrt{1+\beta} \\
-\sqrt{-\beta} & -\sqrt{1+\beta} & 0
\end{array}\right)
$$

where $\beta=\alpha_{(1)} / \alpha_{(3)}$.

2. Bosonic Neumann matrices appearing in the exponential of the kinematical part of the superstring vertex are defined as [26, 30]:

$$
\begin{aligned}
& \bar{N}_{m n}^{r s}=\delta^{r s} \delta_{m n}-2 \sqrt{\frac{\omega_{m(r)} \omega_{n(s)}}{m n}}\left(A^{(r) T} \Gamma^{-1} A^{(s)}\right)_{m n}, \\
& \bar{N}_{m 0}^{r s}=-\sqrt{2 \mu \alpha_{s} \omega_{m(r)}} \epsilon_{0}^{s t} \alpha_{t} \bar{N}_{m}^{r}, \quad s \in\{1,2\}, \\
& \bar{N}_{00}^{r s}=(1-4 \mu \alpha K)\left(\delta^{r s}+\frac{\sqrt{\alpha_{r} \alpha_{s}}}{\alpha_{3}}\right), \quad r, s \in\{1,2\}, \\
& \bar{N}_{00}^{r 3}=\delta^{r 3}-\sqrt{-\frac{\alpha_{r}}{\alpha_{3}}}, \quad r \in\{1,2\} .
\end{aligned}
$$

where $\alpha=a_{(1)} \alpha_{(2)} \alpha_{(3)}$ and the matrices $A^{(r)}, \Gamma, \bar{N}_{m}^{r}$ and $\mathrm{K}$ are defined below.

$$
\begin{aligned}
\Gamma & =\sum_{r=1}^{3} A^{(r)} U_{(r)} A^{(r) T}, \\
U_{(r)} & =C^{-1}\left(C_{(r)}-\mu \alpha_{r}\right), \quad C_{m n}=m \delta_{m n}, \\
\left(C_{(r)}\right)_{m n} & =\omega_{m(r)} \delta_{m n}=\sqrt{n^{2}+\left(\alpha_{(r)} \mu\right)^{2}} \delta_{m n}, \\
\bar{N}^{r} & =-C^{-1 / 2} A^{(r) T} \Gamma^{-1} B, \quad K=-\frac{1}{4} B^{T} \Gamma^{-1} B .
\end{aligned}
$$


where the matrices $A^{(r)}$ and $B$ for $m, n>0$ can be obtained from the Fourier transforming the kinematical constraints in eqn.(3.17)-(3.19):

$$
\begin{aligned}
A_{m n}^{(1)} & =(-1)^{n} \frac{2 \sqrt{m n} \beta}{\pi} \frac{\sin m \pi \beta}{m^{2} \beta^{2}-n^{2}}, \\
A_{m n}^{(2)} & =\frac{2 \sqrt{m n}(\beta+1)}{\pi} \frac{\sin m \pi \beta}{m^{2}(\beta+1)^{2}-n^{2}}, \\
A_{m n}^{(3)} & =\delta_{m n}, \\
B_{m} & =-\frac{2}{\pi} \frac{\alpha_{3}}{\alpha_{1} \alpha_{2}} m^{-3 / 2} \sin m \pi \beta,
\end{aligned}
$$

with $\beta=\alpha_{(1)} / \alpha_{(3)}$. Below we define the matrices $X^{(r)}$ appearing in Fourier expansion of constraints in eqn.(4.18). For $r=1,2$, we have [26]:

$$
\begin{aligned}
X_{m n}^{(r)} & =\left(C^{1 / 2} A^{(r)} C^{-1 / 2}\right)_{m n} & & \text { if } m, n>0, \\
& =\frac{\alpha_{(3)}}{\alpha_{(r)}}\left(C^{-1 / 2} A^{(r)} C^{1 / 2}\right)_{-m,-n}, & & \text { if } m, n<0, \\
& =-\frac{1}{\sqrt{2}} \epsilon^{r s} \alpha_{(s)}\left(C^{1 / 2} B\right)_{m} & & \text { if } n=0 \text { and } m>0, \\
& =1 & & \text { if } m=n=0, \\
& =0 & & \text { otherwise. }
\end{aligned}
$$

with $X_{m n}^{(3)}=\delta_{m n}$.

3. Neumann matrices with negative indices are related to the ones with positive indices as: $\bar{N}_{-m,-n}^{r s}$. They are related to $\bar{N}_{m n}^{r s}$ via $[26,30]$ :

$$
\bar{N}_{-m,-n}^{r s}=-\left(U_{(r)} \bar{N}^{r s} U_{(s)}\right)_{m n}, \quad m, n>0
$$

Further, the following factorization theorem would be useful in verifying that the kinematical vertex satisfies the kinematic constraints given in eqn.(4.16) and (4.17) [29, 30]:

$$
\bar{N}_{m n}^{r s}=-(1-4 \mu \alpha K)^{-1} \frac{\alpha}{\alpha_{r} \omega_{n(s)}+\alpha_{s} \omega_{m(r)}}\left[U_{(r)}^{-1} C_{(r)}^{1 / 2} C \bar{N}^{r}\right]_{m}\left[U_{(s)}^{-1} C_{(s)}^{1 / 2} C \bar{N}^{s}\right]_{n}(
$$

4. The fermionic Neumann matrices are related to the bosonic ones as [30]:

$$
\begin{aligned}
Q_{m n}^{r s} & =e\left(\alpha_{r}\right) \sqrt{\left|\frac{\alpha_{s}}{\alpha_{r}}\right|}\left[P_{(r)}^{-1} U_{(r)} C^{1 / 2} \bar{N}^{r s} C^{-1 / 2} U_{(s)} P_{(s)}^{-1}\right]_{m n}, \\
Q_{n}^{r} & =\frac{e\left(\alpha_{r}\right)}{\sqrt{\left|\alpha_{r}\right|}}(1-4 \mu \alpha K)^{-1}(1-2 \mu \alpha K(1+\Pi))\left[P_{(r)} C_{(r)}^{1 / 2} C^{1 / 2} \bar{N}^{r}\right]_{n} .
\end{aligned}
$$


with,

$$
P_{n(r)} \equiv \frac{1-\rho_{n(r)} \Pi}{\sqrt{1-\rho_{n(r)}^{2}}} .
$$

Explicitely in our case:

$$
\begin{aligned}
Q_{m n}^{r s \dot{\alpha} \dot{\beta}} & \left.=\sqrt{\alpha_{r} \alpha_{s}}\left[P_{(r)}^{-1}\right] U_{(r)} C^{1 / 2} \bar{N}^{r s} C^{-1 / 2} U_{(s)} P_{(s)}^{-1}\right]_{m n}^{\dot{\alpha} \dot{\beta}}, \\
Q_{n}^{r \dot{\alpha} \dot{\beta}} & =i \sqrt{2} \frac{\alpha}{\sqrt{\left|\alpha_{r}\right|}}(1-4 \mu \alpha K)^{-1}\left[P_{(r)} C_{(r)}^{1 / 2} C^{1 / 2} \bar{N}^{r}\right]_{n}^{\dot{\alpha} \dot{\beta}},
\end{aligned}
$$

and

$$
\begin{aligned}
Q_{m n}^{r s \alpha \beta} & =\sqrt{\alpha_{r} \alpha_{s}}\left[P_{(r)} U_{(r)} C^{1 / 2} \bar{N}^{r s} C^{-1 / 2} U_{(s)} P_{(s)}\right]_{m n}^{\alpha \beta}, \\
Q_{n}^{r \alpha \beta} & =i \sqrt{2} \frac{\alpha}{\sqrt{\left|\alpha_{r}\right|}}\left[P_{(r)}^{-1} C_{(r)}^{1 / 2} C^{1 / 2} \bar{N}^{r}\right]_{n}^{\alpha \beta},
\end{aligned}
$$

where,

$$
P_{n(r)} \equiv \frac{1-\rho_{n(r)}}{\sqrt{1-\rho_{n(r)}^{2}}} .
$$

\section{References}

[1] J. Maldacena, The large $N$ limit of superconformal field theories and supergravity, Adv. Theor. Math. Phys. 2 (1998) 231, [hep-th/9711200]; S. S. Gubser, I. R. Klebanov and A. M. Polyakov, Gauge theory correlators from non-critical string theory, Phys. Lett. B 428 (1998) 105, [hep-th/9802109]; E. Witten, Anti-de Sitter space and holography, Adv. Theor. Math. Phys. 2 (1998) 253, [hep-th/9802150]; O. Aharony, S. S. Gubser, J. Maldacena, H. Ooguri and Y. Oz, Large $N$ field theories, string theory and gravity, Phys. Rept. 323 (2000) 183, [hep-th/9905111] and references therein.

[2] D. Berenstein, J. Maldacena and H. Nastase, Strings in flat space and pp waves from $\mathcal{N}$ = 4 super Yang Mills, JHEP 0204 (2002) 013, [hep-th/0202021].

[3] R. Penrose, "Any space-time has a plane wave as a limit," in Differential geometry and relativity, pp. 271-275, Reidel, Dordrecht, 1976.

[4] R. Güven, "Plane wave limits and T-duality," Phys. Lett. B482 (2000) 255, [hepth/0005061].

[5] M.Blau, J.Figuero-O'Farrill and G. Papadopoulos, "Penrose limits, supergravity and brane dynamics", Class. Quant. Grav. 19 (2002) 4753, [hep-th/0202111]. 
[6] M.Blau, J.Figuero-O'Farrill, C. Hull and G. Papadopoulos, " A new maximally supersymmetric background of IIB superstring theory," JHEP 0201 (2000) 047, [hep-th/0110242]; M.Blau, J.Figuero-O'Farrill and G. Papadopoulos, "Penrose limits and maximal supersymmetry," Class. Quant. Grav. 19 (2000) L87, [hep-th/0201081].

[7] R.R.Metsaev, "Type IIB Green-Schwarz superstring in plane wave Ramond-Ramond background",Nucl. Phys. B625 (2002) 70, [hep-th/0112044]; R.R. Metsaev, A.A. Tseytlin, "Exactly solvable model of superstring in Ramond-Ramond plane wave background", Phys.Rev. D65 (2002) 126004, [hep-th/0202109].

[8] C. Kristjansen, J. Plefka, G. W. Semenoff and M. Staudacher, "A new double-scaling limit of $N=4$ super Yang-Mills theory and PP-wave strings," Nucl. Phys. B 643 (2002) 3, [hep-th/0205033].

[9] N. R. Constable, D. Z. Freedman, M. Headrick, S. Minwalla, L. Motl, A. Postnikov and W. Skiba, "PP-wave string interactions from perturbative Yang-Mills theory," JHEP 0207 (2002) 017, [hep-th/0205089].

[10] R. Gopakumar, "String interactions in PP-waves," Phys. Rev. Lett. 89 (2002) 171601, [hep-th/0205174].

[11] N. Beisert, C. Kristjansen, J. Plefka, G. W. Semenoff and M. Staudacher, BMN correlators and operator mixing in $N=4$ super Yang-Mills theory, Nucl. Phys. B 650 (2003) 125, [hepth/0208178].

[12] A. Santambrogio and D. Zanon, "Exact anomalous dimensions of $N=4$ Yang-Mills operators with large R charge," Phys. Lett. B 545 (2002) 425, [hep-th/0206079].

[13] D. J. Gross, A. Mikhailov and R. Roiban, "A calculation of the plane wave string Hamiltonian from $N=4$ super-Yang-Mills theory," [hep-th/0208231].

[14] J. Gomis, S. Moriyama and J. Park, "SYM description of SFT Hamiltonian in a pp-wave background," [hep-th/0210153].

[15] J. G. Zhou, "pp-wave string interactions from string bit model," Phys. Rev. D 67 (2003) 026010, [hep-th/0208232].

[16] D. Vaman and H. Verlinde, "Bit Strings from N=4 Gauge Theory," [hep-th/0209215].

[17] Y. j. Kiem, Y. b. Kim, S. m. Lee and J. m. Park, "pp-wave / Yang-Mills correspondence: An explicit check," Nucl. Phys. B 642 (2002) 389, [hep-th/0205279].

[18] D. Berenstein and H. Nastase, "On lightcone string field theory from super Yang-Mills and holography," [hep-th/0205048]. 
[19] M. x. Huang, "Three point functions of $N=4$ super Yang Mills from light cone string field theory in pp-wave," Phys. Lett. B 542 (2002) 255, [hep-th/0205311].

[20] C. S. Chu, V. V. Khoze and G. Travaglini, "Three-point functions in $N=4$ Yang-Mills theory and pp-waves," JHEP 0206 (2002) 011, [hep-th/0206005].

[21] P. Lee, S. Moriyama and J. w. Park, "Cubic interactions in pp-wave light cone string field theory," Phys. Rev. D 66 (2002) 085021, [hep-th/0206065].

[22] C. S. Chu, V. V. Khoze and G. Travaglini, "pp-wave string interactions from n-point correlators of BMN operators," JHEP 0209 (2002) 054, [hep-th/0206167].

[23] J. Pearson, M. Spradlin, D. Vaman, H. Verlinde and A. Volovich, "Tracing the string: $B M N$ correspondence at finite $J^{2} / N, "[$ hep-th/0210102].

[24] U. Gursoy, "Predictions for pp-wave string amplitudes from perturbative SYM," [hepth/0212118].

[25] N. Beisert, C. Kristjansen, J. Plefka and M. Staudacher, "BMN gauge theory as a quantum mechanical system," Phys. Lett. B 558 (2003) 229, [hep-th/0212269].

[26] M. Spradlin and A. Volovich, "Superstring interactions in a pp-wave background," Phys. Rev. D 66 (2002) 086004, [hep-th/0204146].

[27] M. Spradlin and A. Volovich, "Superstring interactions in a pp-wave background. II," JHEP 0301 (2003) 036, [hep-th/0206073].

[28] I. R. Klebanov, M. Spradlin and A. Volovich, "New effects in gauge theory from pp-wave superstrings," Phys. Lett. B 548 (2002) 111, [hep-th/0206221].

[29] J. H. Schwarz, "Comments on superstring interactions in a plane-wave background," JHEP 0209 (2002) 058, [hep-th/0208179].

[30] A. Pankiewicz, "More comments on superstring interactions in the pp-wave background," JHEP 0209 (2002) 056, [hep-th/0208209].

[31] A. Pankiewicz and B. Stefanski, "pp-wave light-cone superstring field theory," [hepth/0210246].

[32] Y. H. He, J. H. Schwarz, M. Spradlin and A. Volovich, "Explicit formulas for Neumann coefficients in the plane-wave geometry," [hep-th/0211198].

[33] A. Karch and L. Randall, "Open and closed string interpretation of SUSY CFT's on branes with boundaries," JHEP 0106 (2001) 063, [hep-th/0105132]. 
[34] O. DeWolfe, D. Z. Freedman and H. Ooguri, "Holography and defect conformal field theories," Phys. Rev. D 66 (2002) 025009, [hep-th/0111135].

[35] C. Bachas, J. de Boer, R. Dijkgraaf and H. Ooguri, "Permeable conformal walls and holography," JHEP 0206 (2002) 027, [hep-th/0111210].

[36] P. Lee, H. Ooguri and J. w. Park, "Boundary states for AdS(2) branes in AdS(3)," Nucl. Phys. B 632 (2002) 283, [hep-th/0112188].

[37] B. Ponsot, V. Schomerus and J. Teschner, "Branes in the Euclidean AdS(3)," JHEP 0202 (2002) 016, [hep-th/0112198].

[38] J. Erdmenger, Z. Guralnik and I. Kirsch, "Four-dimensional superconformal theories with interacting boundaries or defects," [hep-th/0203020].

[39] P. Lee and J. w. Park, "Open strings in PP-wave background from defect conformal field theory," Phys. Rev. D 67 (2003) 026002, [hep-th/0203257].

[40] K. Skenderis and M. Taylor, "Branes in AdS and pp-wave spacetimes," JHEP 0206 (2002) 025, [hep-th/0204054].

[41] O. Bergman, M. R. Gaberdiel and M. B. Green, "D-brane interactions in type IIB planewave background," [hep-th/0205183].

[42] C. S. Chu and P. M. Ho, "Noncommutative D-brane and open string in pp-wave background with B-field," Nucl. Phys. B 636 (2002) 141, [hep-th/0203186].

[43] A. Dabholkar and S. Parvizi, "Dp branes in pp-wave background," Nucl. Phys. B 641 (2002) 223, [hep-th/0203231].

[44] A. Kumar, R. R. Nayak and Sanjay, "D-brane solutions in pp-wave background," Phys. Lett. B 541 (2002) 183, [hep-th/0204025].

[45] M. Alishahiha and A. Kumar, "D-brane solutions from new isometries of pp-waves," Phys. Lett. B 542 (2002) 130, [hep-th/0205134].

[46] Y. Michishita, "D-branes in NSNS and RR pp-wave backgrounds and S-duality," JHEP 0210 (2002) 048, [hep-th/0206131].

[47] M. Cvetic, H. Lu and C. N. Pope, "Penrose limits, pp-waves and deformed M2-branes," [hep-th/0203082]; P. Bain, P. Meessen and M. Zamaklar, "Supergravity solutions for Dbranes in Hpp-wave backgrounds," Class. Quant. Grav. 20 (2003) 913, [hep-th/0205106]; H. Singh, "M5-branes with 3/8 Supersymmetry in PP-wave background," [hep-th/0205020]; S. S. Pal, "Solution to worldvolume action of D3 brane in pp-wave background", Mod. Phys. 
Lett. A 17 (2002) 1735, [hep-th/0205303]; A. Biswas, A. Kumar and K. L. Panigrahi, " $p$ - p' branes in pp-wave background," Phys. Rev. D 66 (2002) 126002, [hep-th/0208042]; M. Cvetic, H. Lu, C. N. Pope and K. S. Stelle, "Linearly-realised worldsheet supersymmetry in pp-wave background," [hep-th/0209193]; R. R. Nayak, D-branes at angle in pp-wave background, [hep-th/0210230]; J. Kumar and A. Rajaraman, "New supergravity solutions for branes in $A d S(3) x S^{* * 3}$," [hep-th/0212145]; L. F. Alday and M. Cirafici, "An example of localized D-branes solution on pp-wave backgrounds," [hep-th/0301253]; K. L. Panigrahi and Sanjay, "D-branes in pp-wave spacetime with nonconstant NS-NS flux," [hepth/0303182]; Sonia Stanciu, Jos Figueroa-O'Farrill, "Penrose limits of Lie Branes and a Nappi-Witten braneworld," [hep-th/0303212].

[48] D. Berenstein, E. Gava, J. M. Maldacena, K. S. Narain and H. Nastase, "Open strings on plane waves and their Yang-Mills duals," [hep-th/0203249].

[49] V. Balasubramanian, M. x. Huang, T. S. Levi and A. Naqvi, "Open strings from $N=4$ super Yang-Mills," JHEP 0208 (2002) 037, [hep-th/0204196].

[50] K. Skenderis and M. Taylor, "Open strings in the plane wave background. I: Quantization and symmetries," [hep-th/0211011]; J. w. Kim, B. H. Lee and H. S. Yang, "Superstrings and D-branes in plane wave," [hep-th/0302060].

[51] K. Skenderis and M. Taylor, "Open strings in the plane wave background. II: Superalgebras and spectra," [hep-th/0212184].

[52] S. Mandelstam, Interacting string picture of dual resonance models, Nucl. Phys. B 64 (1973) 205; ibid., Dual - resonance models, Phys. Rept. 13 (1974) 259; E. Cremmer and L. Gervais, Combining and splitting relativistic strings, Nucl. Phys. B 76 (1974) 209; ibid., Infinite component field theory of interacting relativistic strings and dual theory, Nucl. Phys. B 90 (1975) 410; M. Kaku and K. Kikkawa, The field theory of relativistic strings, pt. 1: Trees, Phys. Rev. D 10 (1974) 1110; ibid., The field theory of relativistic strings. pt. 2: Loops and pomerons, Phys. Rev. D 10 (1974) 1823; S. Mandelstam, Interacting string picture of the Neveu-Schwarz-Ramond model, Nucl. Phys. B 69 (1974) 77.

[53] M. B. Green and J. H. Schwarz, "Superstring Interactions," Nucl. Phys. B 218 (1983) 43.

[54] M. B. Green, J. H. Schwarz and L. Brink, "Superfield Theory of Type II Superstrings," Nucl. Phys. B 219 (1983) 437.

[55] M. B. Green and J. H. Schwarz, "Superstring Field Theory," Nucl. Phys. B 243 (1984) 475.

[56] G. Bonelli, "On type II strings in exact superconformal non-constant RR backgrounds," JHEP 0301 (2003) 065, [hep-th/0301089]. 
[57] M. B. Green, J. H. Schwarz and E. Witten, "Superstring Theory," (Cambridge University Press,Cambridge, 1987) Volume 1, page 288.

[58] B. Stefanski, Jr., "Open String Plane Wave Light Cone Superstring Field Theory," [hepth/0304114]. 